\title{
TRANSFORMATIONS OF CONJUGATE SYSTEMS WITH EQUAL
}

\section{POINT INVARIANTS*}

BY

\section{LUTHER PFAHLER EISENHART}

In a recent memoir $\dagger$ we have established a transformation of a surface $S$ into a surface $S_{1}$ such that the lines joining corresponding points form a congruence whose developables meet $S$ and $S_{1}$ in conjugate systems with equal tangential invariants; we have called it a transformation $\Omega$. In the present paper we consider a transformation of a surface $S$ into a surface $S_{1}$ such that the developables of the congruence of joins of corresponding points meet $S$ and $S_{1}$ in conjugate systems with equal point invariants. In both cases the fundamental operation is the transformation of Moutard $\ddagger$ of an equation of the Laplace type with equal invariants, just as this transformation lies at the base of the various types of transformations in which the two surfaces are the focal sheets of a $W$-congruence. We identify the transformation under discussion with one which is involved in part in a theorem established from a different point of view by Koenigs, who at the time was considering an entirely different problem.§ We make a detailed study of this transformation,\| because it seems to be the foundation of certain important transformations which have been established in other ways and it points the way to other important transformations. The transformations $K$ admit a theorem of permutability just as the other two general types of transformations referred to above. So far as we know every class of transformations of surfaces of a particular kind into suriaces of the same kind is reducible to one of these three types. Consequently we not only know the basis for the existence of a theorem of permutability in the known particular transformations, but in subsequent investigations we shall have a more immediate method of establishing such a theorem for transformations under consideration. An example of this is given at the close of the memoir.

* Presented to the Society, April 25, 1914.

$\dagger$ Conjugate systems with equal tangential invariants and the transformation of Moutard, Rendiconti del circolo matematico di Palermo, vol. 38.

$\ddagger$ Journal de l'école polytechnique, cahier 45 (1878), p. 1; cf. also, Darboux, Leçons, vol. 2, p. 145.

§Comptes Rendus, vol. 113 (1891), p. 1022.

$\|$ Referred to in the following pages as a transformation $K$. 
We show that transformations $K$ are commutative with certain radial transformations, under which the points of a surface are transformed along lines which are concurrent. These transformations enable us to deduce the general theorem of permutability for transformations $K$ from a special case in which the four surfaces consist of two pairs of associate surfaces, that is, two surfaces corresponding with parallelism of tangent planes and such that to asymptotic lines on either correspond a conjugate system on the other.

Although the treatment of $\S \S 1-5$ is stated in terms of ordinary space, the results are equally true in $n$-space, if we substitute for a surface referred to a conjugate system a reseau, as defined by Guichard* and use the term congruence in the restricted sense given to it by this author.

The remainder of the memoir deals with ordinary space. It is shown that each pair of surfaces in the relation of a transformation $K$ gives rise to four surfaces $\Sigma, \Sigma_{1}, \Sigma_{2}, \Sigma^{\prime}$, such that the pairs of surfaces $\Sigma \Sigma_{1}, \Sigma \Sigma_{2}, \Sigma_{1} \Sigma^{\prime}, \Sigma_{2} \Sigma^{\prime}$ are the focal surfaces of $W$-congruences, and conversely, when four surfaces are so related there exist four pairs of surfaces each pair of which are in the relation of a transformation $K$.

In order to apply the transformations $K$ to particular types of surfaces it is desirable to put the equations in the form developed in $\S 9$. The memoir contains one application, namely the solution of the problem of determining when $S$ and $S_{1}$ envelop a two-parameter family of spheres. It is shown that in this case $S$ and $S_{1}$ are isothermic surfaces in the relation of the transformation discovered by Darboux $\dagger$ and studied at length by Bianchi. $\ddagger$ The theorem of permutability of these transformations is an immediate consequence of our general theorem.

The closing section of the memoir contains the solution of the problem under what conditions does the congruence of joins of corresponding points on two surfaces in the relation of a transformation $K$ possess other pairs of points which describe surfaces similarly related. It is shown that, if a congruence possesses more than one pair of such points, it possesses an infinity of pairs.

In a subsequent memoir we shall consider the case where the joins of corresponding points on two surfaces $S$ and $S_{1}$ form a normal congruence and we shall show that the surfaces orthogonal to such a congruence admit a transformation of the Ribaucour type.

* Annales de l'école normale supérieure, sér. 3, vol. 14 (1897), pp. 467-516.

†Annales de l'école normale supérieure, sér. 3, vol. 16 (1899), pp. 491-508.

† Annali di Matematica, ser. 3, vol. 11 (190k. Fp. 83-158. 


\section{Fundamental transformation}

By definition if $x, y, z$ are the cartesian coördinates of a surface $S$ referred to a conjugate system with equal point invariants, these functions are solutions of an equation of the form

$$
\frac{\partial^{2} \theta}{\partial u \partial v}+\frac{\partial \log \sqrt{\rho}}{\partial v} \frac{\partial \theta}{\partial u}+\frac{\partial \log \sqrt{\rho}}{\partial u} \frac{\partial \theta}{\partial v}=0,
$$

where $\rho$ is in general a function of $u$ and $v$.

In accordance with the transformation of Moutard*, if $\theta$ is any solution of equation (1), the function $\xi$ defined by the equations

$$
\frac{\partial \xi}{\partial u}=-\rho\left(x \frac{\partial \theta}{\partial u}-\theta \frac{\partial x}{\partial u}\right), \quad \frac{\partial \xi}{\partial v}=\rho\left(x \frac{\partial \theta}{\partial v}-\theta \frac{\partial x}{\partial v}\right)
$$

is the solution of another equation with equal invariants. In fact, if we differentiate the first of (2) with respect to $v$, we find readily that $\xi$ is a solution of the equation

$$
\frac{\partial^{2} \theta^{\prime}}{\partial u \partial v}-\frac{\partial \log \sqrt{\rho} \theta}{\partial v} \frac{\partial \theta^{\prime}}{\partial u}-\frac{\partial \log \sqrt{\rho} \theta}{\partial u} \frac{\partial \theta^{\prime}}{\partial v}=0 .
$$

If in (2) we replace $\xi$ and $x$ by the quantities $\eta$ and $y$ respectively, and also by $\zeta$ and $z$, the functions $\eta$ and $\zeta$ so defined satisfy equation (3).

When the functions $\xi, \eta, \zeta$ are replaced by $\lambda x_{1}, \lambda y_{1}, \lambda z_{1}$, the necessary and sufficient condition that the surface $S_{1}$, whose coördinates are $x_{1}, y_{1}, z_{1}$, is referred to a conjugate system is that $\lambda$ be a solution of equation (3). We assume that $\lambda$ is such a function and write equations (2) in the form

(4) $\frac{\partial}{\partial u}\left(\lambda x_{1}\right)=-\rho\left(x \frac{\partial \theta}{\partial u}-\theta \frac{\partial x}{\partial u}\right), \quad \frac{\partial}{\partial v}\left(\lambda x_{1}\right)=\rho\left(x \frac{\partial \theta}{\partial v}-\theta \frac{\partial x}{\partial v}\right)$.

It is readily shown that $x_{1}, y_{1}, z_{1}$ are solutions of

$$
\frac{\partial^{2} \phi}{\partial u \partial v}+\frac{\partial}{\partial v} \log \frac{\lambda}{\sqrt{\rho} \theta} \cdot \frac{\partial \phi}{\partial u}+\frac{\partial}{\partial u} \log \frac{\lambda}{\sqrt{\rho} \theta} \cdot \frac{\partial \phi}{\partial v}=0 .
$$

Hence the parametric conjugate system on $S_{1}$ has equal point invariants.

We denote by $M$ and $M_{1}$ corresponding points on $S$ and $S_{1}$ and by $x_{0}, y_{0}, z_{0}$ the coördinates of a point $M_{0}$ on the line joining $M$ and $M_{1}$. Evidently

(6) $x_{0} \pm x+t\left(x_{1}-x\right), \quad y_{0}=y+t\left(y_{1}-y\right), \quad z_{0}=z+t\left(z_{1}-z\right)$,

where $t$ is a function of $u$ and $v$.

By means of equations (4) the first derivatives of $x_{0}$ assume the form

${ }^{*}$ Loc. cit.

Trans. Am. Math. Soc. 2y 


$$
\begin{array}{r}
\frac{\partial x_{0}}{\partial u}=\left[t\left(\frac{\rho \theta}{\lambda}-1\right)+1\right] \frac{\partial x}{\partial u}+\left(x_{1}-x\right)\left(\frac{\partial t}{\partial u}-t \frac{\partial \log \lambda}{\partial u}\right) \\
-\frac{x t}{\lambda}\left(\frac{\partial \lambda}{\partial u}+\rho \frac{\partial \theta}{\partial u}\right), \\
\frac{\partial x_{0}}{\partial v}=\left[1-t\left(1+\frac{\rho \theta}{\lambda}\right)\right] \frac{\partial x}{\partial v}+\left(x_{1}-x\right)\left(\frac{\partial t}{\partial v}-t \frac{\partial \log \lambda}{\partial t}\right) \\
-\frac{x t}{\lambda}\left(\frac{\partial \lambda}{\partial v}-\rho \frac{\partial \theta}{\partial v}\right) .
\end{array}
$$

It is our purpose to show that $\lambda$ can be so chosen that the developables of the congruence $(G)$ of lines joining corresponding points $M$ and $M_{1}$ meet the surfaces $S$ and $S_{1}$ in the parametric conjugate systems with equal point invariants.

In the first place we observe that 1 is a solution of equation (1) and consequently it follows from (2), on replacing $x$ by 1 , that the equations

$$
\partial \lambda / \partial u=-\rho \partial \theta / \partial u, \quad \partial \lambda / \partial v=\rho \partial \theta / \partial v
$$

are consistent and that the function $\lambda$ so defined is a solution of equation (3).

If we substitute this value of $\lambda$ in equations (7) and give to $t$ either of the values

$$
t_{1}=\lambda /(\lambda-\rho \theta), \quad t_{2}=\lambda_{/}^{\prime}(\lambda+\rho \theta),
$$

we obtain respectively

$$
\frac{\partial x_{0} / \partial u}{x_{1}-x}=\frac{\partial y_{0} / \partial u}{y_{1}-y}=\frac{\partial z_{0} / \partial u}{z_{1}-z}, \quad \frac{\partial x_{0} / \partial v}{x_{1}-x}=\frac{\partial y_{0} / \partial v}{y_{1}-y}=\frac{\partial z_{0} / \partial v}{z_{1}-z}
$$

Hence the parametric ruled surfaces of the congruence $(G)$ are developable.

Furthermore, if $F_{1}$ and $F_{2}$ denote the focal points whose coördinates are given by (6) when $t$ is replaced by $t_{1}$ and $t_{2}$ from (9), it is readily shown that $M$ and $M_{1}$ are harmonic to $F_{1}$ and $F_{2}$.

For the function $\lambda$ given by equations (8) the equations (4) assume the form

(10) $\frac{\partial x_{1}}{\partial u}=\frac{\rho}{\lambda}\left[\left(x_{1}-x\right) \frac{\partial \theta}{\partial u}+\theta \frac{\partial x}{\partial u}\right], \quad \frac{\partial x_{1}}{\partial v}=-\frac{\rho}{\lambda}\left[\left(x_{1}-x\right) \frac{\partial \theta}{\partial v}+\theta \frac{\partial x}{\partial v}\right]$.

The foregoing results are stated in

Theorem 1. When a surface $S$ referred to a conjugate system with equal point invariants is known, and also any solution $\theta$ of the point equation of $S$, the coördinates of a surface $S_{1}$ can be found by quadratures (10), and this surface $S_{1}$ is such that the developables of the congruence of lines joining corresponding points on $S$ and $S_{1}$ meet these surfaces in conjugate systems with equal point invariants. Moreover, the focal points of the congruence are hrirmonic to the corresponding points on $S$ and $S_{1}$. 
From (10) it follows that the necessary and sufficient condition that the tangents to the parametric curves on $S$ and $S_{1}$ at corresponding points be parallel is that $\theta$ be constant. From equations (8) it follows that $\lambda$ also must be constant. If we take $\theta=-\lambda=1$, equations (10) become

$$
\frac{\partial x_{1}}{\partial u}=-\rho \frac{\partial x}{\partial u}, \quad \frac{\partial x_{1}}{\partial v}=\rho \frac{\partial x}{\partial v} .
$$

These equations show that in this case $S$ and $S_{1}$ are associate surfaces. ${ }^{*}$ Moreover, it is known that the corresponding conjugate systems on any two associate surfaces have equal point invariants and their equations are of the form (11). Hence the problem of associate surfaces is a special case of the general problem which we are considering.

\section{The theorem of Koenigs. The transformations $K$}

In this section we show that by means of equations (10) we obtain the most general surface $S_{1}$ such that the congruence of lines joining corresponding points of $S$ and $S_{1}$ possess the properties indicated in Theorem 1 . We begin by looking upon the congruence $(G)$ as composed of the tangents to the curves $v=$ const. on a surface $S_{0}$, whose coördinates are $x_{0}, y_{0}, z_{0}$ and on which the parametric system is conjugate.

Lucien Levy $\dagger$ has shown that the cartesian coördinates $x, y, z$ of any point $M$ of a line of the congruence $(G)$ which describes a surface $S$ cut in a conjugate system by the developables of $(G)$ are expressib.e in the form

$$
x=x_{0}-\frac{\sigma}{\frac{\partial \sigma}{\partial u}} \frac{\partial x_{0}}{\partial u}, \quad y=y_{0}-\frac{\sigma}{\frac{\partial \sigma}{\partial u}} \frac{\partial y_{0}}{\partial u}, \quad z=z_{0}-\frac{\sigma}{\frac{\partial \sigma}{\partial u}} \frac{\partial z_{0}}{\partial u},
$$

where $\sigma$ is a solution of the point equation of $S_{0}$, namely

$$
\frac{\partial^{2} \sigma}{\partial u \partial v}+a \frac{\partial \sigma}{\partial u}+b \frac{\partial \sigma}{\partial v}=0
$$

Moreover, each solution of this equation leads to such a surface $S$.

We consider a second surface $S_{1}$ with coördinates $x_{1}, y_{1}, z_{1}$ given by

$$
x_{1}=x_{0}-\frac{\sigma_{1}}{\frac{\partial \sigma_{1}}{\partial u}} \frac{\partial x_{0}}{\partial u}, \quad y_{1}=y_{0}-\frac{\sigma_{1}}{\frac{\partial \sigma_{1}}{\partial u}} \frac{\partial y_{0}}{\partial u}, \quad z_{1}=z_{0}-\frac{\sigma_{1}}{\frac{\partial \sigma_{1}}{\partial u}} \frac{\partial z_{0}}{\partial u},
$$

$\sigma_{1}$ being another solution of (13).

*E. p. 380. A reference of this kind is to the author's Differential Geometry, Ginn and Company, Boston (1909).

$\dagger$ Sur quelques équations linéaires aux derivées partielles, J o u r nal d e l'é c o l e pol yt e chnique, cahier 56 (1886), p. 77. 
For the sake of brevity we put

$$
\begin{array}{cl}
M=a+\frac{1}{\sigma} \frac{\partial \sigma}{\partial v}, & N=b+\frac{1}{\sigma} \frac{\partial \sigma}{\partial u}, \\
h=\frac{\partial a}{\partial u}+a b, & k=\frac{\partial b}{\partial v}+a b .
\end{array}
$$

Thus $h$ and $k$ are the invariants of equation (13). It follows from (13) and $\left(14^{\prime}\right)$ that

$$
\frac{\partial M}{\partial u}=h-M N, \quad \frac{\partial N}{\partial v}=k-M N .
$$

In accordance with the theory of the Laplace transformation* of equation (13) the coördinates of the other focal point of the congruence $(G)$ are of the form

$$
x_{0}^{\prime}=x_{0}+\frac{1}{b} \frac{\partial x_{0}}{\partial u} .
$$

The condition that the focal points be harmonic with respect to the corresponding points on $S$ and $S_{1}$, whose coördinates are given by (12) and (14) is

$$
N_{1}+N=0 \text {, }
$$

where. $N_{1}$ is given by $\left(14^{\prime}\right)$ when $\sigma$ is replaced by $\sigma_{1}$. If we express the condition that $N_{1}$ satisfies an equation analogous to the second of (15), we have

$$
M_{1}=M-2 k / N
$$

where $M_{1}$ is given by $\left(14^{\prime}\right)$ when $\sigma$ is replaced by $\sigma_{1}$. If we require that $M_{1}$ satisfies an equation analogous to the first of (15) we get

$$
\frac{\partial N}{\partial u}=N \frac{\partial \log k}{\partial u}+\frac{N^{2}}{k}(M N-k) \text {. }
$$

Whenever equations (13) and (18) admit a common solution $\sigma$, this function and $\sigma_{1}$, which follows by quadratures from (16) and (17), determine two surfaces $S$ and $S$ in the relation of a transformation $K$.

Later $(\$ 11)$ we shall consider the conditions under which equations (13) and (18) possess a common solution. Now we are interested in showing that when these conditions are satisfied the coördinates of the two surfaces are in the relations expressed by equations (10).

From (12) we obtain by differentiation

$$
\begin{aligned}
& \frac{\partial x}{\partial u}=\sigma\left(\frac{\partial \sigma}{\partial u}\right)^{-2}\left(\frac{\partial^{2} \sigma}{\partial u^{2}} \frac{\partial x_{0}}{\partial u}-\frac{\partial \sigma}{\partial u} \frac{\partial x_{0}}{\partial v}\right) \\
& \frac{\partial x}{\partial v}=\sigma\left(\frac{\partial \sigma}{\partial u}\right)^{-2} N\left(-\frac{\partial \sigma}{\partial v} \frac{\partial x_{0}}{\partial u}+\frac{\partial \sigma}{\partial u} \frac{\partial x_{0}}{\partial v}\right)
\end{aligned}
$$

*E. p. 404. 
The expression for $\partial^{2} x / \partial u \partial v$ obtained from either of these equations can be put in the form

$$
\frac{\partial^{2} x}{\partial u \partial v}=\left(\frac{\partial \sigma}{\partial u}\right)^{-1}\left(N \frac{\partial \sigma}{\partial v} \frac{\partial x}{\partial u}+\frac{\frac{\partial \sigma}{\partial u}\left(\frac{\partial b}{\partial u}-b^{2}\right)-b \frac{\partial^{2} \sigma}{\partial u^{2}}}{N} \frac{\partial x}{\partial v}\right) .
$$

A necessary and sufficient condition that this equation be of the form (1) is that there exist a function $\rho$ defined by

$$
\begin{aligned}
& \frac{\partial \log \sqrt{\rho}}{\partial u}=-\left(\frac{\partial \sigma}{\partial u}\right)^{-1} N \frac{\partial \sigma}{\partial v} \\
& \frac{\partial \log \sqrt{\rho}}{\partial v}=-\frac{\frac{\partial b}{\partial u}-b^{2}-b\left(\frac{\partial \sigma}{\partial u}\right)^{-1} \frac{\partial^{2} \sigma}{\partial u^{2}}}{N} .
\end{aligned}
$$

It is readily shown that these equations are consistent if equation (18) is satisfied. Owing to the fact that the relation between $S$ and $S_{1}$ is reciprocal, it follows that the parametric system on $S_{1}$ also is conjugate with equal point invariants. Hence we have the theorem of Koenigs:*

Theorem 2. If two surfaces $S$ and $S_{1}$ are so related that the focal points of the congruence of lines joining corresponding points $M$ and $M_{1}$ on these surfaces are harmonic with respect to these points, and also if the developables of the congruence meet $S$ and $S_{1}$ in conjugate systems, the latter have equal point invariants.

Now we show that coördinates the of $S$ and $S_{1}$ are in the relation of equations (10).

Since $\sigma_{1}$ determined by quadratures from (16) and (17) is a solution of equation (13), it is evident from (12) that the function $\theta$ given by

$$
\theta=\sigma_{1}-\frac{\sigma}{\frac{\partial \sigma}{\partial u}} \frac{\partial \sigma_{1}}{\partial u}=2 \sigma_{1}\left(\begin{array}{r}
1+b \frac{\sigma}{\partial \sigma} \\
\frac{\partial u}{u}
\end{array}\right)
$$

is a solution of equation (20).

The first derivatives of $\theta$ are given by equations obtained from equations (19) on replacing $x_{0}$ by $\sigma_{1}$; and from these same equations on substituting $\sigma_{1}$ for $\sigma$ we get the first derivatives of $x_{1}$. When these values are substituted in equations (10), they are satisfied, provided that

$$
\lambda=\rho\left(\sigma_{1}-\frac{\sigma}{\frac{\partial \sigma}{\partial u}} \frac{\partial \sigma_{1}}{\partial u}\right) \frac{\sigma}{\partial \sigma} \frac{\partial \sigma_{1}}{\partial u} / \sigma_{1}=\rho \frac{\theta}{\sigma_{1}}\left(\sigma_{1}-\theta\right) .
$$

When this expression for $\lambda$ is substituted in equations (8), we are led to equations (21). Hence we have

\footnotetext{
${ }^{*}$ Loc. cit.
} 
Theorem 3. The transformation (10) of a surface $S$ into a surface $S_{1}$ is the most general case of pairs of surfaces in the relation of Koenigs.

In view of this result we shall hereafter say that $S_{1}$ given by (10) is obtained from $S$ by a transformation $K$.

\section{Theorem of Permutability}

Suppose that we have two solutions $\theta_{1}$ and $\theta_{2}$ of equation (1) and consider the two surfaces $S_{1}$ and $S_{2}$ which arise from $S$ by transformations $K_{1}$ and $K_{2}$ determined by $\theta_{1}$ and $\theta_{2}$ respectively. The coördinates $x_{1}, y_{1}, z_{1}$ and $x_{2}, y_{2}, z_{2}$ are given by equations of the form (10), namely

$$
\begin{aligned}
& \frac{\partial x_{i}}{\partial u}=\frac{\rho}{\lambda_{i}}\left[\left(x_{i}-x\right) \frac{\partial \theta_{i}}{\partial u}+\theta_{i} \frac{\partial x}{\partial u}\right], \\
& \frac{\partial x_{i}}{\partial v}=-\frac{\rho}{\lambda_{i}}\left[\left(x_{i}-x\right) \frac{\partial \theta_{i}}{\partial v}+\theta_{i} \frac{\partial x}{\partial v}\right]
\end{aligned}
$$

where, in accordance with (8),

$$
\frac{\partial \lambda_{i}}{\partial u}=-\rho \frac{\partial \theta_{i}}{\partial u}, \quad \frac{\partial \lambda_{i}}{\partial v}=\rho \frac{\partial \theta_{i}}{\partial v} \quad(i=1,2) .
$$

From (5) it follows that the functions $x_{i}, y_{i}, z_{i}$, defined by (24) and similar equations in $y_{i}$ and $z_{i}$, satisfy the corresponding equation

$$
\frac{\partial^{2} \phi_{i}}{\partial u \partial v}+\frac{\partial}{\partial v} \log \frac{\lambda_{i}}{\sqrt{\rho} \theta_{i}} \frac{\partial \phi_{i}}{\partial u}+\frac{\partial}{\partial u} \log \frac{\lambda_{i}}{\sqrt{\rho} \theta_{i}} \frac{\partial \phi_{i}}{\partial v}=0 \quad(i=1,2) .
$$

It is evident from (4) that these equations are satisfied also by the functions $\theta_{1}^{\prime}$ and $\theta_{2}^{\prime}$ respectively, defined by

$$
\begin{aligned}
& \frac{\partial}{\partial u}\left(\theta_{i}^{\prime} \lambda_{i}\right)=-\rho\left(\theta_{i} \frac{\partial \theta_{j}}{\partial u}-\theta_{j} \frac{\partial \theta_{i}}{\partial u}\right), \\
& \frac{\partial}{\partial v}\left(\theta_{i}^{\prime} \lambda_{i}\right)=\rho\left(\theta_{i} \frac{\partial \theta_{j}}{\partial v}-\theta_{j} \frac{\partial \theta_{i}}{\partial v}\right) \\
& \left(\begin{array}{l}
i=1,2, \quad i \neq j) \\
j=1,2 ;
\end{array}\right.
\end{aligned}
$$

As thus defined each of the functions $\theta_{i}^{\prime}$ is determined only to within the additive function $c_{i} / \lambda_{i}$, where $c_{i}$ is an arbitrary constant. Hereafter in using pairs of functions $\theta_{1}^{\prime}$ and $\theta_{2}^{\prime}$ simultaneously we understand that the constants $c_{i}$ are so determined that

$$
\theta_{1}^{\prime} \lambda_{1}+\theta_{2}^{\prime} \lambda_{2}=0,
$$

which evidently is possible because of the form of (27).

It is our purpose to show that, if we use the functions $\theta_{1}^{\prime}$ and $\theta_{2}^{\prime}$ to determine transformations $K_{1}^{\prime}$ and $K_{2}^{\prime}$ of $S_{1}$ and $S_{2}$ respectively, the resulting surfaces coincide, giving a surface $S^{\prime}$ with coördinates $x^{\prime}, y^{\prime}, z^{\prime}$. 
In the first place we observe that if we put

$$
\rho_{i}=\lambda_{i}^{2} / \rho \theta_{i}^{2},
$$

equations (26) may be given a form similar to (1). Hence for the transformations $K_{1}^{\prime}$ and $K_{2}^{\prime}$ the equations analogous to (25) are

$$
\frac{\partial \lambda_{i}^{\prime}}{\partial u}=-\frac{\lambda_{i}^{2}}{\rho \theta_{i}^{2}} \frac{\partial \theta_{i}^{\prime}}{\partial u}, \quad \frac{\partial \lambda_{i}^{\prime}}{\partial v}=\frac{\lambda_{i}^{2}}{\rho \theta_{i}^{2}} \frac{\partial \theta_{i}^{\prime}}{\partial v} \quad(i=1,2) .
$$

In order that the transforms of $S_{1}$ and $S_{2}$ may coincide, equations analogous to (24) necessitate the conditions

$$
\begin{aligned}
& \frac{\partial x^{\prime}}{\partial u}=\frac{\rho_{1}}{\lambda_{1}^{\prime}}\left[\left(x^{\prime}-x_{1}\right) \frac{\partial \theta_{1}^{\prime}}{\partial u}+\theta_{1}^{\prime} \frac{\partial x_{1}}{\partial u}\right]=\frac{\rho_{2}}{\lambda_{2}^{\prime}}\left[\left(x^{\prime}-x_{2}\right) \frac{\partial \theta_{2}^{\prime}}{\partial u}+\theta_{2}^{\prime} \frac{\partial x_{2}}{\partial u}\right], \\
& \frac{\partial x^{\prime}}{\partial v}=-\frac{\rho_{1}}{\lambda_{1}^{\prime}}\left[\left(x^{\prime}-x_{1}\right) \frac{\partial \theta_{1}^{\prime}}{\partial v}+\theta_{1}^{\prime} \frac{\partial x_{1}}{\partial v}\right]=-\frac{\rho_{2}}{\lambda_{2}^{\prime}}\left[\left(x^{\prime}-x_{2}\right) \frac{\partial \theta_{2}^{\prime}}{\partial v}+\theta_{2}^{\prime} \frac{\partial x_{2}^{\prime}}{\partial v}\right] .
\end{aligned}
$$

If we substitute in the second equation of the first row the expressions for $\partial \theta_{1}^{\prime} / \partial u$ and $\partial x_{i} / \partial u$ given by (24) and (27), we obtain

$$
\left(\theta_{2} \frac{\partial \theta_{1}}{\partial u}-\theta_{1} \frac{\partial \theta_{2}}{\partial u}\right) P+\frac{\lambda_{1} \theta_{1}^{\prime}}{\theta_{2}}\left(\frac{1}{\lambda_{1}^{\prime} \theta_{1}}+\frac{1}{\lambda_{2}^{\prime} \theta_{2}}\right)\left[\left(x^{\prime}-x\right) \frac{\partial \theta_{2}}{\partial u}+\theta_{2} \frac{\partial x}{\partial u}\right]=0
$$

where for the sake of brevity we have put

$$
P=x^{\prime}\left[\frac{\lambda_{1}}{\lambda_{1}^{\prime} \theta_{1}^{2} \theta_{2}}\left(\theta_{1}^{\prime}+\theta_{2}\right)+\frac{\lambda_{2}}{\lambda_{2}^{\prime} \theta_{2}^{\prime}}\right]-\left(\frac{x_{1} \lambda_{1}}{\lambda_{1}^{\prime} \theta_{1}^{2}}+\frac{x_{2} \lambda_{2}}{\lambda_{2}^{\prime} \theta_{2}^{2}}\right)-\frac{\lambda_{1} \theta_{1}^{\prime} x}{\theta_{1}^{2} \theta_{2} \lambda_{1}^{\prime}} .
$$

In a similar manner we obtain from the second row of (31) the equation

(34) $\left(\theta_{2} \frac{\partial \theta_{1}}{\partial v}-\theta_{1} \frac{\partial \theta_{2}}{\partial v}\right) P+\frac{\lambda_{1} \theta_{1}^{\prime}}{\theta_{2}}\left(\frac{1}{\lambda_{1}^{\prime} \theta_{1}}+\frac{1}{\lambda_{2}^{\prime} \theta_{2}}\right)\left[\left(x^{\prime}-x\right) \frac{\partial \theta_{2}}{\partial v}+\theta_{2} \frac{\partial x}{\partial v}\right]=0$.

The equations of condition (32) and (34) are satisfied if $P=0$ and if

$$
\lambda_{1}^{\prime} \theta_{1}+\lambda_{2}^{\prime} \theta_{2}=0 \text {. }
$$

We shall show that these conditions are satisfied and lead to the desired result. If we differentiate equation (35) with respect to $u$ and $v$ separately and require that the functions $\lambda_{i}^{\prime}$ shall satisfy (30), we find that we must have

$$
\lambda_{1}^{\prime} \theta_{1}=\lambda_{1}\left(\theta_{1}^{\prime}+\theta_{2}\right)-\lambda_{2} \theta_{1}, \quad \lambda_{2}^{\prime} \theta_{2}=\lambda_{2}\left(\theta_{2}^{\prime}+\theta_{1}\right)-\lambda_{1} \theta_{2} .
$$

In consequence of (28) these equations are consistent with (35) and furthermore these expressions for $\lambda_{i}^{\prime}$ satisfy equations (30).

Because of (36) the equation obtained by equating $P$ to zero is reducible to

$$
\lambda_{1}^{\prime} \theta_{1} x^{\prime}=\theta_{1}^{\prime} \lambda_{1} x+\lambda_{1} \theta_{2} x_{1}-\lambda_{2} \theta_{1} x_{2},
$$


which, in consequence of (28) and (35), is equivalent to

$$
\lambda_{2}^{\prime} \theta_{2} x^{\prime}=\theta_{2}^{\prime} \lambda_{2} x+\lambda_{2} \theta_{1} x_{2}-\lambda_{1} \theta_{2} x_{1} .
$$

With the aid of equations (36) it is shown that the value of $x^{\prime}$ given by (37) satisfies equations (31). Hence not only does $S^{\prime}$ exist satisfying the above requirements, but its coördinates can be found without quadrature after $\theta_{1}^{\prime}$ and $\theta_{2}^{\prime}$ have been obtained by the quadratures (27).

We shall say that four surfaces $S, S_{1}, S_{2}, S^{\prime}$ in the above relation form a quatern. From (37) it is evident that the corresponding points on the four surfaces lie in a plane.

From the form of (27) it is seen that $\theta_{1}^{\prime}$ is determined to within the additive quantity $c / \lambda_{1}$, where $c$ is an arbitrary constant. The first of equations (36) shows that the corresponding change in $\lambda_{1}^{\prime}$ is the addition of $c / \theta_{1}$. Hence if $\theta_{1}^{\prime}$ is a function giving a surface $S^{\prime}$ and if we denote by $S_{c}^{\prime}$ the surface determined by $\theta_{1}^{\prime}+c / \lambda_{1}$, the coördinates $x_{c}^{\prime}, y_{c}^{\prime}, z_{c}^{\prime}$ of $S_{c}^{\prime}$ are expressible in the form

$$
\left(\lambda_{1}^{\prime} \theta_{1}+c\right) x_{c}^{\prime}=\left(\theta_{1}^{\prime} \lambda_{1}+c\right) x+\lambda_{1} \theta_{2} x_{1}-\lambda_{2} \theta_{1} x_{2},
$$

which may be written also

$$
\left(\lambda_{1}^{\prime} \theta_{1}+c\right) x_{c}^{\prime}=c x+\lambda_{1}^{\prime} \theta_{1} x^{\prime} .
$$

Hence all the corresponding points on the family of surfaces $S_{c}^{\prime}$ lie on the line joining $M$ and $M^{\prime}$, and each point divides the segment $M M^{\prime}$ in the ratio $c / \lambda_{1}^{\prime} \theta_{1}$.

The foregoing results may be assembled into

THEOREM 4. If $S_{1}$ and $S_{2}$ are two surfaces arising from $S$ by transformations $K$, there can be found by a quadrature an infinity of surfaces $S^{\prime}$ each of which is in the relation of transformations $K$ with both $S_{1}$ and $S_{2}$. The corresponding points on the suite of surfaces $S^{\prime}$ lie on a line which passes through the corresponding point on $S$ and in the plane determined by the latter point and the corresponding points on $S_{1}$ and $S_{2}$.

We consider in particular the case when $S_{1}$ is an associate surface of $S$. As shown in $\S 1$ this arises when we take $\theta_{1}=-\lambda_{1}=1$. Now equations (27) for $i=1$ become

$$
\frac{\partial \theta_{1}^{\prime}}{\partial u}=\rho \frac{\partial \theta_{2}}{\partial u}, \quad \frac{\partial \theta_{1}^{\prime}}{\partial v}=-\rho \frac{\partial \theta_{2}}{\partial v} .
$$

Comparing these with (25) and making use of (28) we see that

$$
\theta_{1}^{\prime}=-\lambda_{2}+c, \quad \theta_{2}^{\prime}=-1+c / \lambda_{2} .
$$

Hence if we take $c=0$ the surfaces $S_{2}$ and $S^{\prime}$ are associate. In this case 
equation (37) can be written

$$
\theta_{2}\left(x^{\prime}-x_{1}\right)=\lambda_{2}\left(x_{2}-x\right) \text {. }
$$

There ore we have

Theorem 5. If $S$ and $S_{1}$ are two associate surfaces, and if $S_{2}$ is any surface arising from $S$ by a transformation $K$, there exists a surface $S^{\prime}$ associate to $S_{2}$ which is in the relation of a transformation $K$ with $S_{1}$; moreover, the lines joining corresponding points of $S_{1}$ and $S^{\prime}$ and of $S$ and $S_{2}$ are parallel.

\section{ENVELope of the Planes of a QUATERN}

We have just seen that the four corresponding points $M, M_{1}, M_{2}, M^{\prime}$ of the surfaces of a quatern $S, S_{1}, S_{2}, S^{\prime}$ lie in a plane. Since this plane contains the lines $M M_{1}$ and $M M_{2}$ which generate congruences whose developables meet $S$ in a conjugate system, it envelopes a surface upon which the curves corresponding to these developables form a conjugate system, as follows from the general theory of conjugate systems and congruences. ${ }^{*}$ Moreover, if $\Pi$ is the point of the envelope corresponding to the point $M$ on $S$, the tangent at $\Pi$ to one of these curves passes through the focal points $F_{1}^{\prime}$ and $F_{2}^{\prime}$ of the lines $M M_{1}$ and $M M_{2}$ respectively, and the tangent to the other curve passes through the other focal points $F_{1}^{\prime \prime}$ and $F_{2}^{\prime \prime}$. Since the set of points $M, M_{1}$, $F_{1}^{\prime}, F_{11}^{\prime \prime}$ are harmonic and likewise the points $M, M_{2}, F_{2}^{\prime}, F_{2}^{\prime \prime}$, it follows that the line joining $M_{1}$ and $M_{2}$ passes through $\Pi$. As the surfaces $S$ and $S^{\prime}$ bear to $S_{1}$ a relation similar to that of $S_{1}$ and $S_{2}$ to $S$, it follows that the point II lies also on the line $M M^{\prime}$.

If $X, Y, Z$ denote the coördinates of $\Pi$, in order that it be the intersection of the lines $M M^{\prime}$ and $M_{1} M_{2}$, there must exist functions $\sigma$ and $\tau$ such that

$$
X=x+\sigma\left(x^{\prime}-x\right)=x_{1}+\tau\left(x_{2}-x_{1}\right),
$$

and similar expressions for $Y$ and $Z$. If we eliminate $x^{\prime}$ from equation (37) and from the equation formed by the last two members of (41), we obtain an equation of the form $a x+b x_{1}+c x_{2}=0$. Since the same equation is satisfied by the $y$ 's and $z$ 's, the expressions $a, b$, and $c$ must be zero. These equations are consistent in consequence of (36) and give the expressions

$$
\sigma=\frac{\lambda_{1}^{\prime} \theta_{1}}{\lambda_{1}^{\prime} \theta_{1}-\lambda_{1} \theta_{1}^{\prime}}, \quad \tau=-\frac{\lambda_{2} \theta_{1}}{\lambda_{1} \theta_{2}-\lambda_{2} \theta_{1}} .
$$

When these values are substituted in (41), we obtain

$$
X=\frac{\lambda_{1}^{\prime} \theta_{1} x^{\prime}-\theta_{1}^{\prime} \lambda_{1} x}{\lambda_{1}^{\prime} \theta_{1}-\theta_{1}^{\prime} \lambda_{1}}=\frac{\lambda_{1} \theta_{2} x_{1}-\lambda_{2} \theta_{1} x_{2}}{\lambda_{1} \theta_{2}-\lambda_{2} \theta_{1}},
$$

which are consistent because of (37).

\footnotetext{
* Cf. Guichard, loc. cit., p. 491.
} 
From (6) and (9) it follows that the coördinates of the focal points, namely $x_{i}^{\prime}, y_{i}^{\prime}, z_{i}^{\prime}$ and $x_{i}^{\prime \prime}, y_{i}^{\prime \prime}, z_{i}^{\prime \prime}$ for $i=1,2$, are given by expressions of the form

$$
x_{i}^{\prime}=\frac{\lambda_{i} x_{i}-\rho \theta_{i} x}{\lambda_{i}-\rho \theta_{i}}, \quad x_{i}^{\prime \prime}=\frac{\lambda_{i} x_{i}+\rho \theta_{i} x}{\lambda_{i}+\rho \theta_{i}} \quad(i=1,2) .
$$

Hence the direction-parameters of the lines $F_{1}^{\prime} F_{2}^{\prime}, F_{1}^{\prime \prime} F_{2}^{\prime \prime}$ are proportional to expressions of the form

$$
\begin{aligned}
& x_{1} \lambda_{1}\left(\lambda_{2}-\rho \theta_{2}\right)-x_{2} \lambda_{2}\left(\lambda_{1}-\rho \theta_{1}\right)+\rho x\left(\lambda_{1} \theta_{2}-\lambda_{2} \theta_{1}\right), \\
& x_{1} \lambda_{1}\left(\lambda_{2}+\rho \theta_{2}\right)-x_{2} \lambda_{2}\left(\lambda_{1}+\rho \theta_{1}\right)-\rho x\left(\lambda_{1} \theta_{2}-\lambda_{2} \theta_{1}\right),
\end{aligned}
$$

respectively. With the aid of these values it is readily verified that the point whose coördinates are given by (42) is the intersection of the lines $F_{1}^{\prime} F_{2}^{\prime}$ and $F_{1}^{\prime \prime} F_{2}^{\prime \prime}$.

From the second expression (42) for $X$ we obtain by differentiation

$$
\begin{aligned}
& \frac{\partial X}{\partial u}=\left[x_{1} \lambda_{1}\left(\lambda_{2}+\rho \theta_{2}\right)-x_{2} \lambda_{2}\left(\lambda_{1}+\rho \theta_{1}\right)+\rho x\left(\theta_{1} \lambda_{2}-\theta_{2} \lambda_{1}\right)\right] K_{1}, \\
& \frac{\partial X}{\partial v}=\left[x_{1} \lambda_{1}\left(\lambda_{2}-\rho \theta_{2}\right)-x_{2} \lambda_{2}\left(\lambda_{1}-\rho \theta_{1}\right)-\rho x\left(\theta_{1} \lambda_{2}-\theta_{2} \lambda_{1}\right)\right] K_{2},
\end{aligned}
$$

where

$$
K_{1}=\frac{\theta_{2} \frac{\partial \theta_{1}}{\partial u}-\theta_{1} \frac{\partial \theta_{2}}{\partial u}}{\left(\theta_{1} \lambda_{2}-\theta_{2} \lambda_{1}\right)^{2}}, \quad K_{2}=\frac{\theta_{2} \frac{\partial \theta_{1}}{\partial v}-\theta_{1} \frac{\partial \theta_{2}}{\partial v}}{\left(\theta_{1} \lambda_{2}-\theta_{2} \lambda_{1}\right)^{2}} .
$$

Comparing equations (44) and (45), we note that the curves $v=$ const. on the envelope are tangent to the corresponding lines $F_{1}^{\prime \prime} F_{2}^{\prime \prime}$ and the curves $u=$ const. to the lines $F_{1}^{\prime} F_{2}^{\prime}$, as previously remarked from the general theory. As the congruences of lines $M^{\prime} M_{1}$ and $M^{\prime} M_{2}$ bear to the surface $S^{\prime}$ a relation similar to that of the lines $M M_{1}$ and $M M_{2}$ to $S$, the focal points on the lines $M^{\prime} M_{1}$ and $M^{\prime} M_{2}$ lie on the tangents to the curves $v=$ const. or $u=$ const. It follows that the two tangents at a point $\Pi$ are harmonic with respect to the lines $M M^{\prime}$ and $M_{1} M_{2}$ through the point.

In order to find the equation satisfied by the coördinates of $\Pi$, we differentiate the first of equations (45) with respect to $v$. After direct reductions we find that

$$
\begin{aligned}
\frac{\partial^{2} X}{\partial u \partial v}+\frac{K_{2}}{K_{1}}\left[\frac{\partial \log \sqrt{\rho}}{\partial u}+\right. & \left.\frac{\lambda_{2} \frac{\partial \theta_{1}}{\partial u}-\lambda_{1} \frac{\partial \theta_{2}}{\partial u}}{\theta_{1} \lambda_{2}-\theta_{2} \lambda_{1}}\right] \frac{\partial X}{\partial u} \\
& +\frac{K_{1}}{K_{2}}\left[\frac{\partial \log \sqrt{\rho}}{\partial v}+\frac{\lambda_{2} \frac{\partial \theta_{1}}{\partial v}-\lambda_{1} \frac{\partial \theta_{2}}{\partial v}}{\theta_{1} \lambda_{2}-\theta_{2} \lambda_{1}}\right] \frac{\partial X}{\partial v}=0 .
\end{aligned}
$$

It is evident that ordinarily the invariants of this equation are not equal. 
We inquire whether the point $\Pi$ ever coincides with one of the suite defined by equation (39) for all values of $u$ and $v$. If we equate this value of $x_{c}^{\prime}$ to the second expression (42) for $X$, we are led to the condition $\theta_{1}^{\prime} \lambda_{1}=-c$, which by (27) necessitates $\theta_{2} / \theta_{1}=$ const. In this case $S_{1}$ and $S_{2}$ are homothetic.

Gathering together the above results we have

Theorem 6. If $S, S_{1}, S_{2}, S^{\prime}$ are four surfaces of a quatern, the plane $\Pi$ of four corresponding points $M, M_{1}, M_{2}, M^{\prime}$ touches its envelope in the intersection II of the lines $M M^{\prime}$ and $M_{1} M_{2}$; the parametric lines on the envelope form $a$ conjugate system (with invariants ordinarily unequal) whose tangents are harmonic to the lines $M M^{\prime}$ and $M_{1} M_{2}$ and contain the focal points of the lines $M M_{1}$, $M M_{2}, M^{\prime} M_{1}, M^{\prime} M_{2}$.

5. Relations BetWEen tRansformations $K$ AND RAdIAL TRANSFORMATIONS

If $\omega$ is a solution of equation (1) which is linearly independent of $x, y, z$, the surface $\bar{S}$ whose coördinates $\bar{x}, \bar{y}, \bar{z}$ are given by

$$
\bar{x}=x / \omega, \quad \bar{y}=y / \omega, \quad \bar{z}=z / \omega
$$

is referred to a conjugate system with equal point invariants. In fact, it is easily shown that the coördinates of $\bar{S}$ satisfy the equation

$$
\frac{\partial^{2} \bar{\theta}}{\partial u \partial v}+\frac{\partial}{\partial v} \log \sqrt{\rho} \omega \frac{\partial \bar{\theta}}{\partial u}+\frac{\partial}{\partial u} \log \sqrt{\rho} \omega \frac{\partial \bar{\theta}}{\partial v}=0 .
$$

We shall say that $\bar{S}$ is obtained from $S$ by a radial transformation.

If $\theta$ is a function linearly independent of $x, y, z$, and $\omega$, we shall find that the surfaces $S_{1}$ and $\bar{S}_{1}$ resulting from $S$ and $\bar{S}$ by transformations $K$, determined by $\theta$ and $\bar{\theta}$ respectively, where

$$
\bar{\theta}=\theta / \omega,
$$

are in the relation of a radial transformation. In fact, we shall show that the coördinates $x_{1}, y_{1}, z_{1}$ and $\bar{x}_{1}, \bar{y}_{1}, \bar{z}_{1}$ of $S_{1}$ and $\bar{S}_{1}$ respectively are in the relations

$$
\bar{x}_{1}=x_{1} / \omega_{1}, \quad \bar{y}_{1}=y_{1} / \omega_{1}, \quad \bar{z}_{1}=z_{1} / \omega_{1},
$$

where $\omega_{1}$ is given by the quadratures

$$
\frac{\partial \omega_{1}}{\partial u}=\frac{\rho}{\lambda}\left[\left(\omega_{1}-\omega\right) \frac{\partial \theta}{\partial u}+\theta \frac{\partial \omega}{\partial u}\right], \quad \frac{\partial \omega_{1}}{\partial v}=-\frac{\rho}{\lambda}\left[\left(\omega_{1}-\omega\right) \frac{\partial \theta}{\partial v}+\theta \frac{\partial \omega}{\partial v}\right] .
$$

As equations (52) are of the form of (10), it is evident that they are consistent and that $\omega_{1}$ is a solution of the point equation of $S_{1}$, namely (5). 
The equations of the transformation from $\bar{S}$ to $\bar{S}_{1}$, analogous to (10), are

$$
\begin{aligned}
& \frac{\partial \bar{x}_{1}}{\partial u}=\frac{\rho \omega^{2}}{\bar{\lambda}}\left[\left(\frac{x_{1}}{\omega_{1}}-\frac{x}{\omega}\right) \frac{\partial}{\partial u}\left(\frac{\theta}{\omega}\right)+\frac{\theta}{\omega} \frac{\partial}{\partial u}\left(\frac{x}{\omega}\right)\right], \\
& \frac{\partial \bar{x}_{1}}{\partial v}=-\frac{\rho \omega^{2}}{\bar{\lambda}}\left[\left(\frac{x_{1}}{\omega_{1}}-\frac{x}{\omega}\right) \frac{\partial}{\partial v}\left(\frac{\theta}{\omega}\right)+\frac{\theta}{\omega} \frac{\partial}{\partial v}\left(\frac{x}{\omega}\right)\right] .
\end{aligned}
$$

When the value of $\bar{x}_{1}$ from (51) is substituted in (53) and in the reduction equations (52) are used, it is found that the necessary and sufficient condition that these equations be satisfied is that

$$
\bar{\lambda}=\omega_{1} \lambda \text {. }
$$

With the aid of equations (52) it is readily shown that this value of $\bar{\lambda}$ satisfies the equations

$$
\frac{\partial \bar{\lambda}}{\partial u}=-\rho \omega^{2} \frac{\partial}{\partial u}\left(\frac{\theta}{\omega}\right), \quad \frac{\partial \bar{\lambda}}{\partial v}=\rho \omega^{2} \frac{\partial}{\partial v}\left(\frac{\theta}{\omega}\right),
$$

which are analogous to equations (8). Hence we have

THEOREM 7. If two surfaces $S$ and $\bar{S}$ are in the relation of a radial transformation and a transformation $K$ of $S$ is known, it is possible to find by quadratures a transformation $K$ of $\bar{S}$ such that the new surfaces $S_{1}$ and $\bar{S}_{1}$ are in the relation of a radial transformation.

If in particular $\theta=\omega$, it follows from (55) that $\bar{\lambda}$ is constant. When we take $\bar{\lambda}=-1$, equation (54) becomes

$$
\omega_{1}=-1 / \lambda
$$

which we have seen in $\S 3$ is a solution of equation (5). With the aid of equations (8) it can be shown that this value of $\omega_{1}$ satisfies equations (52). Now $\bar{\theta}=1$, consequently $\bar{S}_{1}$ is associate to $\bar{S}$, as shown in $\S 1$. From these results we have

Theorem 8. If a surface $S$ is subjected to a radial transformation determined by a function $\theta$, and if the associate of the resulting surface, determined by the parametric conjugate system, undergoes a radial transformation determined by $-\lambda$, where $\lambda$ is given by the quadratures (8), the new surface $S_{1}$ and $S$ are in the relation of the transformation $K$ determined by $\theta$.

Suppose now that we have a quatern of surfaces $S, S_{1}, S_{2}, S^{\prime}$, expressed in terms of the same functions appearing in $\S 3$. If $\theta_{2}$ determines a radial transformation of $S$, a comparison of equations (52) and (27) reveals the fact that the function $\omega_{1}=-\theta_{1}^{\prime}$. On the other hand $\omega_{2}=-1 / \lambda_{2}$. Again, since the radial transformation function of $S_{1}$ differs only in sign from the function $\theta_{1}^{\prime}$ of the transformation of $S_{1}$ into $S^{\prime}$, it follows that $\omega^{\prime}=1 / \lambda_{1}^{\prime}$. 
Hence the four surfaces $\bar{S}, \bar{S}_{1}, \bar{S}_{2}, \bar{S}^{\prime}$, whose $x$-coördinates are respectively of the form

$$
\bar{x}=x / \theta_{2}, \quad \bar{x}_{1}=-x_{1} / \theta_{1}^{\prime}, \quad \bar{x}_{2}=-\lambda_{2} x_{2}, \quad \bar{x}^{\prime}=\lambda_{1}^{\prime} x^{\prime},
$$

are radial transforms of the surfaces of the original quatern; and these surfaces themselves form a quatern of the special type of Theorem 5 , the surfaces $\bar{S}$ and $\bar{S}_{2}$ being associate and likewise $\bar{S}_{1}$ and $\bar{S}^{\prime}$. The transformation functions of this new quatern are

$$
\begin{gathered}
\bar{\theta}_{1}=\theta_{1} / \theta_{2}, \quad \bar{\lambda}_{1}=-\lambda_{1} \theta_{1}^{\prime} ; \quad \bar{\theta}_{2}=-\bar{\lambda}_{2}=1 ; \quad \bar{\theta}_{1}^{\prime}=-\bar{\lambda}_{1}^{\prime}=-1 ; \\
\bar{\theta}_{2}^{\prime}=\lambda_{1} \theta_{1}^{\prime}, \quad \bar{\lambda}_{2}^{\prime}=-\theta_{1} / \theta_{2} .
\end{gathered}
$$

It is readily shown that these functions and the coördinates (57) satisfy equations analogous to (37) and (38).

In like manner the four surfaces whose coördinates are of the form

$$
x / \theta_{1}, \quad-\lambda_{1} x_{1}, \quad-x_{2} / \theta_{2}^{\prime}, \quad \lambda_{2}^{\prime} x^{\prime}
$$

form a quatern in radial relation to the corresponding surfaces of the other two quaterns.

Accordingly we have

Theorem 9. When a quatern of surfaces is known, two other quaterns can be found without quadratures, each of which consists of two pairs of associate surfaces.

\section{6. $W$-CONGRUENCES ASSOCIATED WITH A TRANSFORMATION $K$}

We consider a surface $S$ and the associate surface $S_{0}$ whose coördinates $x_{0}, y_{0}, z_{0}$ are given by quadratures of the form

$$
\frac{\partial x_{0}}{\partial u}=-\rho \frac{\partial x}{\partial u}, \quad \frac{\partial x_{0}}{\partial v}=\rho \frac{\partial x}{\partial v},
$$

as follows from (11). These coördinates are solutions of

$$
\frac{\partial^{2} \theta_{0}}{\partial u \partial v}=\frac{\partial \log \sqrt{\rho}}{\partial v} \frac{\partial \theta_{0}}{\partial u}+\frac{\partial \log \sqrt{\rho}}{\partial u} \frac{\partial \theta_{0}}{\partial v},
$$

as is readily shown, if we note that $x$ is a solution of equation (1). If we put

$$
\sigma=\theta_{0} / \sqrt{\rho}
$$

the function $\sigma$ is a solution of the equation

$$
\frac{\partial^{2} \sigma}{\partial u \partial v}=\sqrt{\rho} \frac{\partial^{2}}{\partial u \partial v}\left(\frac{1}{\sqrt{\rho}}\right) \sigma .
$$


In accordance with the theory of infinitesimal deformation of surfaces the surface $\Sigma$, whose coördinates $\xi, \eta, \zeta$ are given by quadratures of the form

$$
\frac{\partial \xi}{\partial u}=z_{0} \frac{\partial y}{\partial u}-y_{0} \frac{\partial z}{\partial u}, \quad \frac{\partial \xi}{\partial v}=z_{0} \frac{\partial y}{\partial v}-y_{0} \frac{\partial z}{\partial v},
$$

corresponds to $S$ with orthogonality of linear elements.* For the sake of brevity we say that $\Sigma$ is the ortho-surface of $S$ determined by $S_{0}$.

By means of (59), equations (63) are reducible to

$$
\frac{\partial \xi}{\partial u}=-\frac{1}{\rho}\left(z_{0} \frac{\partial y_{0}}{\partial u}-y_{0} \frac{\partial z_{0}}{\partial u}\right), \quad \frac{\partial \xi}{\partial v}=\frac{1}{\rho}\left(z_{0} \frac{\partial y_{0}}{\partial v}-y_{0} \frac{\partial z_{0}}{\partial v}\right) .
$$

If we put

$$
x_{0}=\sqrt{\rho} \alpha, \quad y_{0}=\sqrt{\rho} \beta, \quad z_{0}=\sqrt{\rho} \gamma,
$$

the functions $\alpha, \beta, \gamma$ are solutions of equation (62) and equations (64) assume the Lelieuvre form

$$
\frac{\partial \xi}{\partial u}=\left(\beta \frac{\partial \gamma}{\partial u}-\gamma \frac{\partial \beta}{\partial u}\right), \quad \frac{\partial \xi}{\partial v}=-\left(\beta \frac{\partial \gamma}{\partial v}-\gamma \frac{\partial \beta}{\partial v}\right)
$$

In like manner there is a surface $S_{10}$ associate to a surface $S_{1}$ resulting from $S$ by a transformation $K$ and its coördinates, $x_{10}, y_{10}, z_{10}$, are given by

$$
\frac{\partial x_{10}}{\partial u}=-\rho_{1} \frac{\partial x_{1}}{\partial u}, \quad \frac{\partial x_{10}}{\partial v}=\rho_{1} \frac{\partial x_{1}}{\partial v},
$$

where, as follows from (29),

$$
\rho_{1}=\lambda^{2} / \rho \theta^{2}
$$

It can readily be shown that the four surfaces $S, S_{0}, S_{1}, S_{10}$ satisfy the requirements of Theorem 5 , so that $S_{0}$ and $S_{10}$ are in the relation of a transformation $K$. In fact, in this case equation (40) becomes

$$
\theta\left(x_{10}-x_{0}\right)=\lambda\left(x_{1}-x\right) .
$$

If the expressions (10) for $\partial x_{1} / \partial u$ and $\partial x_{1} / \partial v$ be substituted in (67) and $\left(x_{1}-x\right)$ be replaced by its value from (69), we have

$$
\frac{\partial}{\partial u}\left(\theta x_{10}\right)=x_{0} \frac{\partial \theta}{\partial u}+\frac{\lambda}{\rho} \frac{\partial x_{0}}{\partial u}, \quad \frac{\partial}{\partial v}\left(\theta x_{10}\right)=x_{0} \frac{\partial \theta}{\partial v}-\frac{\lambda}{\rho} \frac{\partial x_{0}}{\partial v} .
$$

In a manner analogous to (65) we put

$$
x_{10}=\sqrt{\rho_{1}} \alpha_{1}=-\frac{\lambda}{\sqrt{\rho} \theta} \alpha_{1} .
$$

As defined by equations (8), the function $\lambda$ is a solution of equation (60).

\footnotetext{
*E., p. 382 .
} 
Consequently the function $\sigma_{1}$, defined by

$$
\sigma_{1}=\lambda / \sqrt{\rho},
$$

is a solution of (62). In consequence of equations (71), (72), and (8), equations (70) can be written in the form

$$
\frac{\partial}{\partial u}\left(\sigma_{1} \alpha_{1}\right)=-\left(\sigma_{1} \frac{\partial \alpha}{\partial u}-\alpha \frac{\partial \sigma_{1}}{\partial u}\right), \quad \frac{\partial}{\partial v}\left(\sigma_{1} \alpha_{1}\right)=\left(\sigma_{1} \frac{\partial \alpha}{\partial v}-\alpha \frac{\partial \sigma_{1}}{\partial v}\right) .
$$

Moreover, the coördinates $\xi_{1}, \eta_{1}, \zeta$, of $\Sigma_{1}$, the ortho-surface to $S_{1}$ determined by $S_{10}$, are given by the quadratures

$$
\frac{\partial \xi_{1}}{\partial u}=\left(\beta_{1} \frac{\partial \gamma_{1}}{\partial u}-\gamma_{1} \frac{\partial \beta_{1}}{\partial u}\right), \quad \frac{\partial \xi_{1}}{\partial v}=-\left(\beta_{1} \frac{\partial \gamma_{1}}{\partial v}-\gamma_{1} \frac{\partial \beta_{1}}{\partial v}\right) .
$$

From these results we know that $\Sigma$ and $\Sigma_{1}$ can be so placed in space that they are the focal surfaces of a $W$-congruence, and that the following relations hold:

$$
\xi_{1}-\xi=\beta \gamma_{1}-\beta_{1} \gamma, \quad \eta_{1}-\eta=\gamma \alpha_{1}-\gamma_{1} \alpha,
$$

$$
\zeta_{1}-\zeta=\alpha \beta_{1}-\alpha_{1} \beta \text {. }^{*}
$$

Each solution of equation (62) determines a surface associate to $\Sigma$. We denote by $\Sigma_{01}$ the associate surface corresponding to the solution $\sigma_{1}$ given by (72), and by $\Sigma_{02}$ the associate surface determined by the solution

$$
\sigma_{2}=1 / \sqrt{\rho} .
$$

The first of these gives rise to the $W$-congruence whose focal surfaces are $\Sigma$ and $\Sigma_{1}$. The surface $\Sigma_{01}$ determines an ortho-surface to $\Sigma$, namely $\bar{S}$, whose coördinates $\bar{x}, \bar{y}, \bar{z}$ are

$$
\bar{x}=\sigma_{1} \alpha_{1}, \quad \bar{y}=\sigma_{1} \beta_{1}, \quad \bar{z}=\sigma_{1} \gamma_{1},
$$

where $\alpha_{1}, \beta_{1}, \gamma_{1}$ are given by (71). The surface $\bar{S}_{0}$ whose coördinates are

$$
\bar{x}_{0}=\alpha / \sigma_{1}, \quad \bar{y}_{0}=\beta / \sigma_{1}, \quad \bar{z}_{0}=\gamma / \sigma_{1},
$$

is associate to $\bar{S}$ and is an ortho-surface of $\Sigma_{1} . \dagger$

The equation for $\Sigma_{1}$ analogous to (62), namely

$$
\frac{\partial^{2} \bar{\sigma}}{\partial u \partial v}=\sqrt{\rho_{1}} \frac{\partial^{2}}{\partial u \partial v}\left(\frac{1}{\sqrt{\rho_{1}}}\right) \bar{\sigma}
$$

admits the solution $1 / \sigma_{1}$. This solution determines an associate surface of $\Sigma_{1}$ which leads to the ortho-surface $\bar{S}_{0}$ of $\Sigma_{1}$. Moreover, the joins of corresponding points on this associate surface and $\Sigma_{01}$ are concurrent. $\ddagger$

* Cf. E., pp. 417-420.

$\dagger$ Cf. E., p. 420.

$\ddagger$ Cf. Darboux, Leçons, vol. 4, p. 69. 
We observe that $1 / \sqrt{\rho_{1}}$ also is a solution of equation (79). We denote by $\Sigma_{10}$ the surface associate to $\Sigma_{1}$ determined by this solution. If we apply the Moutard transformation of the form $(73)$ to $1 / \sqrt{\rho}$, we obtain $1 / \sqrt{\rho_{1}}$. In fact, it is readily shown that the equations

$$
\begin{aligned}
& \frac{\partial}{\partial u}\left(\sigma_{1} \frac{1}{\sqrt{\rho_{1}}}\right)=-\left(\sigma_{1} \frac{\partial}{\partial u} \frac{1}{\sqrt{\rho}}-\frac{1}{\sqrt{\rho}} \frac{\partial \sigma_{1}}{\partial u}\right), \\
& \frac{\partial}{\partial v}\left(\sigma_{1} \frac{1}{\sqrt{\rho_{1}}}\right)=\left(\sigma_{1} \frac{\partial}{\partial v} \frac{1}{\sqrt{\rho}}-\frac{1}{\sqrt{\rho}} \frac{\partial \sigma_{1}}{\partial v}\right),
\end{aligned}
$$

reduce to equations (8). Making use of results which we have established elsewhere, ${ }^{*}$ we have

Theorem 10. The surfaces $\Sigma_{02}$ and $\Sigma_{10}$, associate to $\Sigma$ and $\Sigma_{1}$, and determined by $1 / \sqrt{\rho}$ and $1 / \sqrt{\rho_{1}}$ respectively, are such that the lines joining corresponding points on these surfaces form a congruence whose developables meet them in the conjugate parametric system, which has equal tangential invariants; and the lines of intersection of the tangent planes to $\Sigma_{02}$ and $\Sigma_{10}$ form a congruence whose developables are parametric and whose focal planes are harmonic to these tangent planes.

We say that two surfaces, related as $\Sigma_{02}$ and $\Sigma_{10}$ are in this theorem, are in the relation of a transformation $\Omega$.

We turn to the consideration of the $W$-congruence, determined by the solution $\sigma_{2}=1 / \sqrt{\rho}$ of equation (62), of which $\Sigma$ is one of the focal surfaces. Since $S$ is the corresponding ortho-surface of $\Sigma$, the functions $\alpha_{2}, \beta_{2}, \gamma_{2}$ for the second sheet $\Sigma_{2}$ are given by the equations

$$
\alpha_{2}=\sqrt{\rho} x, \quad \beta_{2}=\sqrt{\rho} y, \quad \gamma_{2}=\sqrt{\rho} z,
$$

which are analogous to equations (77). These functions are solutions of the equation

$$
\frac{\partial^{2} \sigma^{\prime}}{\partial u \partial v}=\frac{1}{\sqrt{\rho}} \frac{\partial^{2} \sqrt{\rho}}{\partial u \partial v} \cdot \sigma^{\prime},
$$

as is readily shown. Moreover, from (59), (65), and (81) it follows that

$$
\begin{aligned}
& \frac{\partial}{\partial u}\left(\frac{\alpha_{2}}{\sqrt{\rho}}\right)=-\left(\frac{1}{\sqrt{\rho}} \frac{\partial \alpha}{\partial u}-\alpha \frac{\partial}{\partial u} \frac{1}{\sqrt{\rho}}\right), \\
& \frac{\partial}{\partial v}\left(\frac{\alpha_{2}}{\sqrt{\rho}}\right)=\left(\frac{1}{\sqrt{\rho}} \frac{\partial \alpha}{\partial v}-\alpha \frac{\partial}{\partial v} \frac{1}{\sqrt{\rho}}\right),
\end{aligned}
$$

which are of the same form as (73).

In like manner the function $\bar{\sigma}_{1}=1 / \sqrt{\rho_{1}}$ determines a $W$-congruence for

\footnotetext{
* Rendiconti, loc. cit.
} 
which $\Sigma_{1}$ is one of the focal surfaces. Since $S_{1}$ is the corresponding orthosurface to $\Sigma_{1}$, the functions $\alpha^{\prime}, \beta^{\prime}, \gamma^{\prime}$ for the second focal sheet $\Sigma^{\prime}$ are given by

$$
\alpha^{\prime}=\sqrt{\rho_{1}} x_{1}=-\frac{\lambda}{\sqrt{\rho} \theta} x_{1}, \quad \beta^{\prime}=-\frac{\lambda}{\sqrt{\rho} \theta} y_{1}, \quad \gamma^{\prime}=-\frac{\lambda}{\sqrt{\rho} \theta} z_{1} .
$$

It is easily found that the function

$$
\sigma_{2}^{\prime}=\sqrt{\rho} \theta
$$

is a solution of equation (82). If we substitute in equations (10) the expressions for $x_{1}$ and $x$ which follow from equations (81) and (84), the resulting equations may be given the form

$$
\begin{aligned}
& \frac{\partial}{\partial u}\left(\sigma_{2}^{\prime} \alpha^{\prime}\right)=-\left(\sigma_{2}^{\prime} \frac{\partial \alpha_{2}}{\partial u}-\alpha_{2} \frac{\partial \sigma_{2}^{\prime}}{\partial u}\right), \\
& \frac{\partial}{\partial v}\left(\sigma_{2}^{\prime} \alpha^{\prime}\right)=\left(\sigma_{2}^{\prime} \frac{\partial \alpha_{2}}{\partial v}-\alpha_{2} \frac{\partial \sigma_{2}^{\prime}}{\partial v}\right) .
\end{aligned}
$$

Comparing these equations with (73), we observe that the lines joining corresponding points on the surfaces $\Sigma_{2}$ and $\Sigma^{\prime}$ form a $W$-congruence, for which these are the focal surfaces. It remains to find the relations between the coördinates of these surfaces.

From equations (65), (69), (71), (81), and (84), we obtain the relation

$$
\alpha^{\prime}=\alpha+\frac{\lambda}{\rho \theta}\left(\alpha_{1}-\alpha_{2}\right) .
$$

Analogous to equations (75) are the following:

$$
\begin{gathered}
\xi_{2}-\xi=\beta \gamma_{2}-\gamma \beta_{2}, \quad \xi^{\prime}-\xi_{1}=\beta_{1} \gamma^{\prime}-\gamma_{1} \beta^{\prime}, \\
\xi^{\prime}-\xi_{2}=\beta_{2} \gamma^{\prime}-\gamma_{2} \beta^{\prime},
\end{gathered}
$$

which are consistent in view of (87). From these follows also

$$
\xi^{\prime}=\xi+\frac{\lambda}{\rho \theta}\left(\beta_{2} \gamma_{1}-\beta_{1} \gamma_{2}\right) .
$$

From the foregoing results we have

Theorem 11. When a pair of surfaces $S$ and $S_{1}$ in the relation of a transformation $K$ are known, it is possible to find by quadratures four surfaces $\Sigma, \Sigma_{1}$, $\Sigma_{2}, \Sigma^{\prime}$ such that the pairs $\Sigma \Sigma_{1}, \Sigma \Sigma_{2}, \Sigma_{1} \Sigma^{\prime}, \Sigma_{2} \Sigma^{\prime}$ are the focal surfaces of four $W$-congruences and the asymptotic lines on these surfaces correspond.

We say that four such $W$-congruences form a quatern, which we indicate by $W\left(\Sigma, \Sigma_{1}, \Sigma_{2}, \Sigma^{\prime}\right)$.

From this theorem we derive 
Theorem 12. Four surfaces $S, S_{1}, S_{2}, S^{\prime}$ forming a quatern under transformations $K$ determine twelve $W$-congruences forming six quaterns of $W$-congruences.

Let $W\left(\Sigma, \Sigma_{1}, \Sigma_{3}, \Sigma^{\prime}\right)$ and $W\left(\Sigma, \Sigma_{2}, \Sigma_{3}, \Sigma^{\prime \prime}\right)$ be the quaterns determined by $S$ and $S_{1}$ and by $S$ and $S_{2}$ respectively in accordance with Theorem 11 . The pair of surfaces $S_{1}$ and $S^{\prime}$ determine a quatern $W\left(\Sigma_{1}, \Sigma_{4}, \Sigma^{\prime}, \Sigma^{\prime \prime \prime}\right)$. But from the nature of the theorem of permutability it follows that there is a quatern $W\left(\Sigma_{2}, \Sigma_{4}, \Sigma^{\prime \prime}, \Sigma^{\prime \prime \prime}\right)$ determined by $S_{2}$ and $S^{\prime}$. Furthermore, it is readily seen that these focal surfaces are so placed that there exist also the quaterns $W\left(\Sigma, \Sigma_{1}, \Sigma_{2}, \Sigma_{4}\right)$ and $W\left(\Sigma_{3}, \Sigma^{\prime}, \Sigma^{\prime \prime}, \Sigma^{\prime \prime \prime}\right)$.

\section{Transformations $K$ Determined by a QUATERn of $W$-Congruences}

In this section we shall show that every quatern of $W$-congruences gives rise to four pairs of surfaces in the relation of transformations $K$. In doing so we make use of the results of Bianchi* concerning the determination of such quaterns.

Let $\Sigma$ be a surface referred to its asymptotic lines and defined by equations (66), where $\alpha, \beta, \gamma$ are linearly independent solutions of an equation of the form

$$
\frac{\partial^{2} \sigma}{\partial u \partial v}=M \sigma
$$

$M$ being a function of $u$ and $v$ in general. If $\sigma_{1}$ and $\sigma_{2}$ are two independent solutions of this equation, the functions $\alpha_{1}, \beta_{1}, \gamma_{1}$ and $\alpha_{2}, \beta_{2}, \gamma_{2}$, given by quadratures of the form (73), determine two surfaces $\Sigma_{1}$ and $\Sigma_{2}$ whose cartesian coördinates $\xi_{1}, \eta_{1}, \zeta_{1}$ and $\xi_{2}, \eta_{2}, \zeta_{2}$ are expressible in the form (75). Moreover, the functions $\alpha_{i}, \beta_{i}, \gamma_{i}(i=1,2)$ satisfy the equations

$$
\frac{\partial^{2} \bar{\sigma}_{i}}{\partial u \partial v}=\sigma_{i} \frac{\partial^{2}}{\partial u \partial v}\left(\frac{1}{\sigma_{i}}\right) \bar{\sigma}_{i} \quad(i=1,2) .
$$

In accordance with the theorem of Moutard these equations are satisfied also by the functions $\sigma_{1}^{\prime}$ and $\sigma_{2}^{\prime}$, defined by

$$
\begin{array}{lll}
\frac{\partial}{\partial u}\left(\sigma_{1} \sigma_{1}^{\prime}\right)=-\sigma_{1}^{2} \frac{\partial}{\partial u}\left(\frac{\sigma_{2}}{\sigma_{1}}\right), & \frac{\partial}{\partial v}\left(\sigma_{1} \sigma_{1}^{\prime}\right)=\sigma_{1}^{2} \frac{\partial}{\partial v}\left(\frac{\sigma_{2}}{\sigma_{1}}\right), \\
\frac{\partial}{\partial u}\left(\sigma_{2} \sigma_{2}^{\prime}\right)=-\sigma_{2}^{2} \frac{\partial}{\partial u}\left(\frac{\sigma_{1}}{\sigma_{2}}\right), & \frac{\partial}{\partial v}\left(\sigma_{2} \sigma_{2}^{\prime}\right)=\sigma_{2}^{2} \frac{\partial}{\partial v}\left(\frac{\sigma_{1}}{\sigma_{2}}\right) .
\end{array}
$$

In the subsequent discussion we assume that the constants of integration are so chosen that

$$
\sigma_{2} \sigma_{2}^{\prime}=-\sigma_{1} \sigma_{1}^{\prime}
$$

* Lezioni di Geometria Differenziale, Pisa (1903), vol. 2, pp. 71-74. 
in which case we have

$$
\sigma_{1}^{\prime} \frac{\partial^{2}}{\partial u \partial v}\left(\frac{1}{\sigma_{1}^{\prime}}\right)=\sigma_{2}^{\prime} \frac{\partial^{2}}{\partial u \partial v}\left(\frac{1}{\sigma_{2}^{\prime}}\right) .
$$

Bianchi has shown that the functions $\alpha^{\prime}, \beta^{\prime}, \gamma^{\prime}$, defined by

where

$$
\begin{gathered}
\alpha^{\prime}=\alpha+t\left(\alpha_{1}-\alpha_{2}\right), \quad \beta^{\prime}=\beta+t\left(\beta_{1}-\beta_{2}\right), \\
\gamma^{\prime}=\gamma+t\left(\gamma_{1}-\gamma_{2}\right),
\end{gathered}
$$

$$
t=-\frac{\sigma_{2}}{\sigma_{1}^{\prime}}=\frac{\sigma_{1}}{\sigma_{2}^{\prime}}
$$

satisfy equations (86), also the equations

$$
\begin{aligned}
& \frac{\partial}{\partial u}\left(\sigma_{1}^{\prime} \alpha^{\prime}\right)=-\left(\sigma_{1}^{\prime} \frac{\partial \alpha_{1}}{\partial u}-\alpha_{1} \frac{\partial \sigma_{1}^{\prime}}{\partial u}\right), \\
& \frac{\partial}{\partial v}\left(\sigma_{1}^{\prime} \alpha^{\prime}\right)=\left(\sigma_{1}^{\prime} \frac{\partial \alpha_{1}}{\partial v}-\alpha_{1} \frac{\partial \sigma_{1}^{\prime}}{\partial v}\right),
\end{aligned}
$$

and analogous ones for $\beta^{\prime}$ and $\gamma^{\prime}$, and finally also

$$
\frac{\partial^{2} \theta^{\prime}}{\partial u \partial v}=M^{\prime} \theta^{\prime}
$$

where $M^{\prime}$ is the function equal to either member of (95).

These functions $\alpha^{\prime}, \beta^{\prime}, \gamma^{\prime}$ determine a surface $\Sigma^{\prime}$, whose coördinates are given by

$$
\begin{gathered}
\xi^{\prime}=\xi+t\left(\beta_{2} \gamma_{1}-\beta_{1} \gamma_{2}\right), \quad \eta^{\prime}=\eta+t\left(\gamma_{2} \alpha_{1}-\gamma_{1} \alpha_{2}\right), \\
\zeta^{\prime}=\zeta+t\left(\alpha_{2} \beta_{1}-\alpha_{1} \beta_{2}\right),
\end{gathered}
$$

such that $\Sigma_{1}$ and $\Sigma^{\prime}$ are the focal surfaces of a $W$-congruence and likewise $\Sigma_{2}$ and $\Sigma^{\prime}$ of another $W$-congruence. Hence we have a quatern of $W$-congruences $W\left(\Sigma, \Sigma_{1}, \Sigma_{2}, \Sigma^{\prime}\right)$.

We suppose that we have a quatern $W\left(\Sigma, \Sigma_{1}, \Sigma_{2}, \Sigma^{\prime}\right)$ defined in this general way and we introduce three functions $\rho, \lambda, \theta$, defined by the equations

$$
\sigma_{1}=\lambda / \sqrt{\rho}, \quad \sigma_{2}=1 / \sqrt{\rho}, \quad \sigma_{2}^{\prime}=\sqrt{\rho} \theta .
$$

In terms of these functions equation (90) can be given the form (62) and equations (93) reduce to (8). In accordance with (94) we have

$$
\sigma_{1}^{\prime}=-\theta \sqrt{\rho} / \lambda=1 / \sqrt{\rho_{1}},
$$

the function $\sqrt{\rho_{1}}$ being defined by this equation.

In accordance with the general theory of $W$-congruences and infinitesimal 
deformation of surfaces, the surfaces $S$ and $S_{0}$ whose coördinates $x, y, z$ and $x_{0}, y_{0}, z_{0}$ are given by (81) and (65) are ortho-surfaces of $\Sigma$ and $\Sigma_{2}$ respectively. In like manner the surfaces $\bar{S}$ and $\bar{S}_{0}$ whose coördinates $\bar{x}, \bar{y}, \bar{z}$ and $\bar{x}_{0}, \bar{y}_{0}, \bar{z}_{0}$ are of the form (77) and (78) are ortho-surfaces of $\Sigma$ and $\Sigma_{1}$; and $S_{1}$ and $S_{10}$, whose coördinates $x_{1}, y_{1}, z_{1}$ and $x_{10}, y_{10}, z_{10}$ are given by (84) and (71), are ortho-surfaces of $\Sigma_{1}$ and $\Sigma^{\prime}$. Also the quantities $\bar{x}_{1}, \bar{y}_{1}, \bar{z}_{1}$ and $\bar{x}_{10}, \bar{y}_{10}, \bar{z}_{10}$ which are of the form

$$
\bar{x}_{1}=\sqrt{\rho} \theta \alpha^{\prime}, \quad \bar{x}_{10}=\alpha_{2} / \sqrt{\rho} \theta
$$

are the coördinates of ortho-surfaces $\overline{S_{1}}$ and $\overline{S_{10}}$ of $\Sigma_{2}$ and $\Sigma^{\prime}$ respectively.

When the values of $\alpha_{2}$ and $\alpha^{\prime}$ given by (81) and (84) are substituted in (86), we obtain equations (4). Hence the surfaces $S$ and $S_{1}$ are in the relation of a transformation $K$. In like manner it can be shown that each pair of surfaces $\overline{S S}_{1}, \bar{S}_{0} \bar{S}_{10}, S_{0} S_{10}$ are in the relation of a transformation $K$. Moreover, it follows from Theorem 10 that each of the above four pairs determines a pair of surfaces referred to a conjugate system with equal tangential invariants which are in the relation of a transformation $\Omega$, and the spherical representation of this conjugate system on any one of these surfaces is the same as of the asymptotic lines on one of the surfaces $\Sigma, \Sigma_{1}, \Sigma_{2}, \Sigma^{\prime}$. Hence we have

Theorem 13. If $\Sigma, \Sigma_{1}, \Sigma_{2}, \Sigma^{\prime}$ are the focal surfaces of a quatern of $W$ congruences, the eight associated ortho-surfaces of the former can be arranged into four pairs such that the surfaces of each pair are in the relation of a transformation $K$; moreover, each pair determines the quatern of $W$-congruences; also each pair determines two other surfaces which are in the relation of a transformation $\Omega$.

\section{EQUATIONS OF a TRANSFORMATION $K$ IN ANOTHER FORM}

In applying the transformations $K$ to particular types of surfaces whose transforms are to be of the same type, it is frequently advisable to have the equations of the transformation in another form, which we will now establish.

Let $S$ be a surface referred to a conjugate system with equal point invariants, and let $X, Y, Z ; X_{1}, Y_{1}, Z_{1}$ and $X_{2}, Y_{2}, Z_{2}$, denote the direction-cosines of the normal to $S$ and of the bisectors of the angles between the coördinate lines. If one of these angles be denoted by $2 \omega$, we have

$$
\frac{\partial x}{\partial u}=\sqrt{E}\left(\cos \omega X_{1}-\sin \omega X_{2}\right), \quad \frac{\partial x}{\partial v}=\sqrt{G}\left(\cos \omega X_{1}+\sin \omega X_{2}\right),
$$

where $E, F, G$ denote the coefficients of the linear element of $S$. Since 
$F=\sqrt{E G} \cos 2 \omega$, we have

$$
\begin{aligned}
& \frac{\partial X}{\partial u}=-\frac{D}{\sqrt{E}} \frac{1}{\sin 2 \omega}\left(\sin \omega X_{1}-\cos \omega X_{2}\right), \\
& \frac{\partial X}{\partial v}=\frac{D^{\prime \prime}}{\sqrt{G}} \frac{1}{\sin 2 \omega}\left(\sin \omega X_{1}+\cos \omega X_{2}\right),
\end{aligned}
$$

where $D$ and $D^{\prime \prime}$ denote the fundamental coefficients of the second order for $S . *$

If we compare equation (1) with the Gauss equations for $S \dagger$, we observe that

$$
\left\{\begin{array}{l}
12 \\
1
\end{array}\right\}=-\frac{\partial \log \sqrt{\rho}}{\partial v}, \quad\left\{\begin{array}{l}
12 \\
2
\end{array}\right\}=-\frac{\partial \log \sqrt{\rho}}{\partial u}
$$

the Christoffel symbols $\left\{\begin{array}{c}r a \\ t\end{array}\right\}$ being formed with respect to the linear element of $S$. From the definition of these symbols $\ddagger$ it follows that equations (106) lead to the identities

$$
\begin{aligned}
& \frac{\partial \sqrt{E}}{\partial v}=-\sqrt{E} \frac{\partial \log \sqrt{\rho}}{\partial v}-\sqrt{G} \cos 2 \omega \frac{\partial \log \sqrt{\rho}}{\partial u}, \\
& \frac{\partial \sqrt{G}}{\partial u}=-\sqrt{G} \frac{\partial \log \sqrt{\rho}}{\partial u}-\sqrt{E} \cos 2 \omega \frac{\partial \log \sqrt{\rho}}{\partial v} .
\end{aligned}
$$

The expressions for the other Christoffel symbols are as follows:

$$
\begin{aligned}
& \left\{\begin{array}{l}
11 \\
1
\end{array}\right\}=\frac{\partial \log \sqrt{E}}{\partial u}+2 \cot 2 \omega \frac{\partial \omega}{\partial u}-\sqrt{\frac{E}{G}} \cos 2 \omega \frac{\partial \log \sqrt{\rho}}{\partial v}, \\
& \left\{\begin{array}{l}
11 \\
2
\end{array}\right\}=\frac{E}{G} \frac{\partial \log \sqrt{\rho}}{\partial v}-2 \sqrt{\frac{E}{G}} \frac{1}{\sin 2 \omega} \frac{\partial \omega}{\partial u}, \\
& \left\{\begin{array}{c}
22 \\
1
\end{array}\right\}=\frac{G}{E} \frac{\partial \log \sqrt{\rho}}{\partial u}-2 \sqrt{\frac{G}{E}} \frac{1}{\sin 2 \omega} \frac{\partial \omega}{\partial v}, \\
& \left\{\begin{array}{c}
22 \\
2
\end{array}\right\}=\frac{\partial \log \sqrt{G}}{\partial v}+2 \cot 2 \omega \frac{\partial \omega}{\partial v}-\sqrt{\frac{G}{E}} \cos 2 \omega \frac{\partial \log \sqrt{\rho}}{\partial u} .
\end{aligned}
$$

If equations (104) be differentiated and use be made of equation (1) and

*E., p. 116.

$\dagger$ E., p. 154.

$\ddagger$ E., p. 153. 
of the Gauss equations*

$$
\begin{aligned}
& \frac{\partial^{2} x}{\partial u^{2}}=\left\{\begin{array}{c}
11 \\
1
\end{array}\right\} \frac{\partial x}{\partial u}+\left\{\begin{array}{c}
11 \\
2
\end{array}\right\} \frac{\partial x}{\partial v}+D X, \\
& \frac{\partial^{2} x}{\partial v^{2}}=\left\{\begin{array}{c}
22 \\
1
\end{array}\right\} \frac{\partial x}{\partial u}+\left\{\begin{array}{c}
22 \\
2
\end{array}\right\} \frac{\partial x}{\partial v}+D^{\prime \prime} X,
\end{aligned}
$$

we obtain

$$
\begin{aligned}
& \frac{\partial X_{1}}{\partial u}=\frac{D}{2 \sqrt{E} \cos \omega} X+\left(\sqrt{\frac{E}{G}} \frac{\partial \log \sqrt{\rho}}{\partial v} \sin 2 \omega-\frac{\partial \omega}{\partial u}\right) X_{2}, \\
& \frac{\partial X_{1}}{\partial v}=\frac{D^{\prime \prime}}{2 \sqrt{G} \cos \omega} X-\left(\sqrt{\frac{G}{E}} \frac{\partial \log \sqrt{\rho}}{\partial u} \sin 2 \omega-\frac{\partial \omega}{\partial v}\right) X_{2}, \\
& \frac{\partial X_{2}}{\partial u}=\frac{-D}{2 \sqrt{E} \sin \omega} X-\left(\sqrt{\frac{E}{G}} \frac{\partial \log \sqrt{\rho}}{\partial v} \sin 2 \omega-\frac{\partial \omega}{\partial u}\right) X_{1}, \\
& \frac{\partial X_{2}}{\partial v}=\frac{D^{\prime \prime}}{2 \sqrt{G} \sin \omega} X+\left(\sqrt{\frac{G}{E}} \frac{\partial \log \sqrt{\rho}}{\partial u} \sin 2 \omega-\frac{\partial \omega}{\partial v}\right) X_{1} .
\end{aligned}
$$

The conditions of integrability of these equations are satisfied in consequence of the Gauss and Codazzi equations $\dagger$ which in the present case can be given the form

$$
\frac{D D^{\prime \prime}}{\sqrt{E G}} \frac{1}{\sin 2 \omega}=\frac{\partial}{\partial v}\left(\sqrt{\frac{E}{G}} \sin 2 \omega \frac{\partial \log \sqrt{\rho}}{\partial v}\right)
$$

and

$$
+\frac{\partial}{\partial u}\left(\sqrt{\frac{G}{E}} \sin 2 \omega \frac{\partial \log \sqrt{\rho}}{\partial u}\right),
$$

$$
\begin{aligned}
& \frac{\partial D}{\partial v}+\frac{\partial \log \sqrt{\rho}}{\partial v} D+\left(\frac{E}{G} \frac{\partial \log \sqrt{\rho}}{\partial v}-2 \sqrt{\frac{E}{G}} \frac{1}{\sin 2 \omega} \frac{\partial \omega}{\partial u}\right) D^{\prime \prime}=0 \\
& \frac{\partial D^{\prime \prime}}{\partial u}+\frac{\partial \log \sqrt{\rho}}{\partial u} D^{\prime \prime}+\left(\frac{G}{E} \frac{\partial \log \sqrt{\rho}}{\partial u}-2 \sqrt{\frac{G}{E}} \frac{1}{\sin 2 \omega} \frac{\partial \omega}{\partial v}\right) D=0
\end{aligned}
$$

We introduce three functions $a, b, w$ by means of equations of the form

$$
x_{1}-x=\frac{1}{\lambda}\left(a X_{1}+b X_{2}+w X\right),
$$

where $x_{1}, y_{1}, z_{1}$ are the cartesian coördinates of a surface $S_{1}$ in the relation of a transformation $K$ with $S$ and $\lambda$ is the corresponding function defined by

†E., p. 155. 
equations (8). In consequence of (113), the fundamental equations (10) can be written

$$
\begin{aligned}
\frac{\partial x_{1}}{\partial u}=\frac{\rho}{\lambda^{2}}\left[\left(a \frac{\partial \theta}{\partial u}+\theta \lambda \cos \omega \sqrt{E}\right)\right. & X_{1} \\
& \left.+\left(b \frac{\partial \theta}{\partial u}-\theta \lambda \sin \omega \sqrt{E}\right) X_{2}+w \frac{\partial \theta}{\partial u} X\right],
\end{aligned}
$$$$
\frac{\partial x_{1}}{\partial v}=-\frac{\rho}{\lambda^{2}}\left[\left(a \frac{\partial \theta}{\partial v}+\theta \lambda \cos \omega \sqrt{G}\right) X_{1}\right.
$$

$$
\left.+\left(b \frac{\partial \theta}{\partial v}+\theta \lambda \sin \omega \sqrt{G}\right) X_{2}+w \frac{\partial \theta}{\partial v} X\right] .
$$

When the expression (113) for $x_{1}$ is substituted in these equations, we obtain two equations of the form

$$
A_{1} X_{1}+B_{1} X_{2}+C_{1} X=0, \quad A_{2} X_{1}+B_{2} X_{2}+C_{2} X=0,
$$

where $A_{1}, B_{1}, \cdots, C_{2}$ are determinate functions which must be zero, since these equations are satisfied also by the $Y$ 's and $Z$ 's. This gives the following six equations of condition:

$$
\begin{aligned}
& \frac{\partial a}{\partial u}=(\rho \theta-\lambda) \sqrt{E} \cos \omega+b\left(\sqrt{\frac{E}{G}} \frac{\partial \log \sqrt{\rho}}{\partial v} \sin 2 \omega-\frac{\partial \omega}{\partial u}\right) \\
& +\frac{w D}{2 \sqrt{E} \cos \omega} \\
& \frac{\partial a}{\partial v}=-(\rho \theta+\lambda) \sqrt{G} \cos \omega-b\left(\sqrt{\frac{G}{E}} \frac{\partial \log \sqrt{\rho}}{\partial u} \sin 2 \omega-\frac{\partial \omega}{\partial v}\right) \\
& +\frac{w D^{\prime \prime}}{2 \sqrt{G} \cos \omega} \\
& \text { (115) } \frac{\partial b}{\partial u}=-(\rho \theta-\lambda) \sqrt{E} \sin \omega-a\left(\sqrt{\frac{E}{G}} \frac{\partial \log \sqrt{\rho}}{\partial v} \sin 2 \omega-\frac{\partial \omega}{\partial u}\right) \\
& -\frac{w D}{2 \sqrt{E} \sin \omega} \\
& \frac{\partial b}{\partial v}=-(\rho \theta+\lambda) \sqrt{G} \sin \omega+a\left(\sqrt{\frac{G}{E}} \frac{\partial \log \sqrt{\rho}}{\partial u} \sin 2 \omega-\frac{\partial \omega}{\partial v}\right) \\
& +\frac{w D^{\prime \prime}}{2 \sqrt{G} \sin \omega} \\
& \frac{\partial w}{\partial u}=-\frac{D}{2 \sqrt{E}}\left(\frac{a}{\cos \omega}-\frac{b}{\sin \omega}\right), \quad \frac{\partial w}{\partial v}=-\frac{D^{\prime \prime}}{2 \sqrt{G}}\left(\frac{a}{\cos \omega}+\frac{b}{\sin \omega}\right) .
\end{aligned}
$$


With the aid of equations (8), (107), (111), and (112) it can be shown that the conditions of integrability of these equations are satisfied.

If we put for the sake of brevity

$$
T^{2}=a^{2}+b^{2}+w^{2},
$$

it follows from (115) that

$$
\begin{aligned}
& T \frac{\partial T}{\partial u}=(\rho \theta-\lambda) \sqrt{E}(a \cos \omega-b \sin \omega), \\
& T \frac{\partial T}{\partial v}=-(\rho \theta+\lambda) \sqrt{G}(a \cos \omega+b \sin \omega) .
\end{aligned}
$$

If $X^{\prime}, Y^{\prime}, Z^{\prime}$ denote the direction-cosines of the normal to $S_{1}$, we have from (114),

$$
\begin{aligned}
\Delta X^{\prime}=-w \sin \omega( & \left.\sqrt{E} \frac{\partial \theta}{\partial v}+\sqrt{G} \frac{\partial \theta}{\partial u}\right) X_{1} \\
& +w \cos \omega\left(-\sqrt{E} \frac{\partial \theta}{\partial v}+\sqrt{G} \frac{\partial \theta}{\partial u}\right) X_{2} \\
& +X\left[a \sin \omega\left(\sqrt{E} \frac{\partial \theta}{\partial v}+\sqrt{G} \frac{\partial \theta}{\partial u}\right)\right. \\
& \left.+b \cos \omega\left(\sqrt{E} \frac{\partial \theta}{\partial v}-\sqrt{G} \frac{\partial \theta}{\partial u}\right)+\theta \lambda \sqrt{E G} \sin 2 \omega\right],
\end{aligned}
$$

where $\Delta^{2}$ is equal to the sum of the squares of the coefficients of $X_{1}, X_{2}$, and $X$.

9. WhEN $S$ AND $S_{1}$ ENVELOP A TWO-PARAMETER FAMILY OF SPHERES.

Transformations $D_{m}$ OF AN ISOTHERMIC SURFACE

As an example of the foregoing method we consider the case where $S$ and $S_{1}$ constitute the envelope of a two parameter family of spheres. In this case we must have

$$
\begin{gathered}
x_{1}-x=\frac{R \Delta}{\lambda}\left(X^{\prime}-X\right), \quad y_{1}-y=\frac{R \Delta}{\lambda}\left(Y^{\prime}-Y\right), \\
z_{1}-z=\frac{R \Delta}{\lambda}\left(Z^{\prime}-Z\right),
\end{gathered}
$$

where $R \Delta / \lambda$ is the radius of the spheres.

When the expressions for $x_{1}$ and $X^{\prime}$ given by (113) and (118) are substituted in the first of equations (119), and the coefficients of $X_{1}, X_{2}$, and $X$ on 
the two sides of the resulting identity are equated, we get

$$
\begin{aligned}
a & =-w \sin \omega R\left(\sqrt{E} \frac{\partial \theta}{\partial v}+\sqrt{G} \frac{\partial \theta}{\partial u}\right), \\
b & =-w \cos \omega R\left(\sqrt{E} \frac{\partial \theta}{\partial v}-\sqrt{G} \frac{\partial \theta}{\partial u}\right), \\
w= & {\left[a \sin \omega\left(\sqrt{E} \frac{\partial \theta}{\partial v}+\sqrt{G} \frac{\partial \theta}{\partial u}\right)+b \cos \omega\left(\sqrt{E} \frac{\partial \theta}{\partial v}-\sqrt{G} \frac{\partial \theta}{\partial u}\right)\right.} \\
& +\theta \lambda \sqrt{E G} \sin 2 \omega-\Delta] R .
\end{aligned}
$$

By means of the first two of these equations the last is equivalent to

$$
a^{2}+b^{2}+w^{2}=R w(\theta \lambda \sqrt{E G} \sin 2 \omega-\Delta) .
$$

In consequence of the first two of equations (120) equation (118) can be written

$$
\Delta X^{\prime}=\frac{a}{R} X_{1}+\frac{b}{R} X_{2}-X\left[\frac{a^{2}+b^{2}}{R w}-\theta \lambda \sqrt{E G} \sin 2 \omega\right],
$$

whence it follows that

$$
\Delta^{2}=\frac{a^{2}}{R^{2}}+\frac{b^{2}}{R^{2}}+\left(\frac{a^{2}+b^{2}}{R w}-\theta \lambda \sqrt{E G} \sin 2 \omega\right)^{2} .
$$

Eliminating $\Delta$ from (121) and (123), we obtain

$$
a^{2}+b^{2}+w^{2}=2 w \theta \lambda \sqrt{E G} \sin 2 w R,
$$

and consequently from (121) and (124) we have

$$
\Delta=-\theta \lambda \sqrt{E G} \sin 2 \omega .
$$

By means of (120), (121), (125) we obtain the following equations:

$$
\begin{aligned}
& \sqrt{E}(a \cos \omega-b \sin \omega)=-w \sin 2 \omega \sqrt{E G} \frac{\partial \theta}{\partial u} R=-\frac{T^{2}}{2 \theta \lambda} \frac{\partial \theta}{\partial u}, \\
& \sqrt{G}(a \cos \omega+b \sin \omega)=-w \sin 2 \omega \sqrt{E G} \frac{\partial \theta}{\partial v} R=-\frac{T^{2}}{2 \theta \lambda} \frac{\partial \theta}{\partial v} .
\end{aligned}
$$

With the aid of these expressions and (8) equations (117) are reducible to

$$
\begin{aligned}
& \frac{\partial \log }{\partial u} \frac{T^{2}}{=}=\left(\frac{1}{\theta}-\frac{\rho}{\lambda}\right) \frac{\partial \theta}{\partial u}=\frac{\partial}{\partial u} \log (\lambda \theta), \\
& \frac{\partial \log T^{2}}{\partial v}=\left(\frac{1}{\theta}+\frac{\rho}{\lambda}\right) \frac{\partial \theta}{\partial v}=\frac{\partial}{\partial v} \log (\lambda \theta) .
\end{aligned}
$$


Hence on integration we have

$$
T^{2}=2 c \lambda \theta
$$

where $c$ denotes an arbitrary constant.

Substituting in (126) the value (127) of $T^{2}$, we get

$$
\begin{aligned}
& \sqrt{E}(a \cos \omega-b \sin \omega)=-c \frac{\partial \theta}{\partial u}, \\
& \sqrt{G}(a \cos \omega+b \sin \omega)=-c \frac{\partial \theta}{\partial v} .
\end{aligned}
$$

If these equations be differentiated with respect to $v$ and $u$ respectively, and in the reduction use be made of the preceding equations, we find

$$
(\lambda+\rho \theta) \sqrt{G} \cos 2 \omega=0, \quad(\lambda-\rho \theta) \sqrt{E} \cos 2 \omega=0 .
$$

Hence we must have $2 \omega=\pi / 2$, that is, the parametric curves are the lines of curvature. From (107) it follows that

$$
\sqrt{E}=V / \sqrt{\rho}, \quad \sqrt{G}=U / \sqrt{\rho},
$$

where $U$ and $V$ are functions of $u$ and $v$ alone respectively, and consequently $S$ is an isothermic surface. As the relation between $S$ and $S_{1}$ is reciprocal, it follows that $S_{1}$ also is isothermic. We shall show that there exist pairs of isothermic surfaces satisfying these equations.

Suppose that $S$ is an isothermic surface. The parameters of its lines of curvature can be chosen so that

$$
\sqrt{E}=\sqrt{G}=1 / \sqrt{\rho}=e^{\phi},
$$

where $\phi$ is a function thus defined. Now in the above equations

$$
\sin \omega=\cos \omega=1 / \sqrt{2}, \quad D=e^{2 \phi} / \rho_{1}, \quad D^{\prime \prime}=e^{2 \phi} / \rho_{2},
$$

where $\rho_{1}$ and $\rho_{2}$ are the principal radii of curvature of $S$. If we put

$$
\alpha=\frac{a-b}{\sqrt{2}}, \quad \beta=\frac{a+b}{\sqrt{2}}, \quad c=-\frac{1}{m},
$$

and replace $\theta$ and $\lambda$ by $m \theta$ and $m \lambda$, which evidently is consistent with equations (8), equations (115), (128), and (8) are equivalent to

$$
\frac{\partial \alpha}{\partial u}=-m \lambda e^{\phi}+m e^{-\phi} \theta-\frac{\partial \phi}{\partial v} \beta+\frac{w e^{\phi}}{\rho_{1}}, \quad \frac{\partial \alpha}{\partial v}=\beta \frac{\partial \phi}{\partial u},
$$




$$
\begin{aligned}
& \frac{\partial \beta}{\partial u}=\alpha \frac{\partial \phi}{\partial v}, \quad \frac{\partial \beta}{\partial v}=-m \lambda e^{\phi}-m e^{-\phi} \theta-\frac{\partial \phi}{\partial u} \alpha+\frac{w e^{\phi}}{\rho_{2}}, \\
& \frac{\partial w}{\partial u}=-\alpha \frac{e^{\phi}}{\rho_{1}}, \quad \frac{\partial w}{\partial v}=-\beta \frac{e^{\phi}}{\rho_{2}}, \\
& \frac{\partial \theta}{\partial u}=e^{\phi} \alpha, \quad \frac{\partial \theta}{\partial v}=e^{\phi} \beta, \quad \frac{\partial \lambda}{\partial u}=-e^{-\phi} \alpha, \quad \frac{\partial \lambda}{\partial v}=e^{-\phi} \beta .
\end{aligned}
$$

Furthermore equation (127) becomes

$$
\alpha^{2}+\beta^{2}+w^{2}+2 \lambda \theta m=0 .
$$

It is readily shown that the first derivatives of the left hand member of equation (133) are zero, and consequently by choosing the initial values properly we can find solutions of equations (132) satisfying (133). It should be mentioned that all the conditions of integrability of (132) are easily shown to be satisfied.

If in the subsequent discussion $X_{1}, Y_{1}, Z_{1}$ and $X_{2}, Y_{2}, Z_{2}$ denote the direction-cosines of the tangents to the curves $v=$ const. and $u=$ const. respectively, it is necessary and sufficient to replace $X_{1}$ and $X_{2}$ in the foregoing formulas by $\left(X_{1}+X_{2}\right) / \sqrt{2}$ and $\left(-X_{1}+X_{2}\right) / \sqrt{2}$ respectively, as is evident from (104).

With these changes equations (113) and (114) become

$$
x_{1}-x=\frac{1}{\lambda m}\left(\alpha X_{1}+\beta X_{2}+w X\right)
$$

and

$$
\begin{aligned}
& \frac{\partial x_{1}}{\partial u}=\frac{e^{-\phi}}{\lambda^{2} m}\left[\left(\alpha^{2}+\theta \lambda m\right) X_{1}+\alpha \beta X_{2}+\alpha w X\right], \\
& \frac{\partial x_{1}}{\partial v}=-\frac{e^{-\phi}}{\lambda^{2} m}\left[\alpha \beta X_{1}+\left(\beta^{2}+\theta \lambda m\right) X_{2}+\beta w X\right] .
\end{aligned}
$$

From these equations we have for the fundamental coefficients $E_{1}, F_{1}, G_{1}$, of the surface $S_{1}$ the expressions

$$
E_{1}=G_{1}=\frac{e^{-2 \phi} \theta^{2}}{\lambda^{2}}, \quad F_{1}=0
$$

which proves that $S_{1}$ also is isothermic.

For this case the direction-cosines $X^{\prime}, Y^{\prime}, Z^{\prime}$ of the normal to $S_{1}$, as given for the general case by (118), are of the form

$$
X^{\prime}=\frac{1}{\theta \lambda m}\left[\left(w^{2}+\theta \lambda m\right) X+\alpha w X_{1}+\beta w X_{2}\right]
$$


With the aid of this expression equation (134) may be written

$$
x_{1}+\frac{\theta}{w} X^{\prime}=x+\frac{\theta}{w} X,
$$

which shows that $\theta / w$ is the radius of the sphere tangent to $S$ and $S_{1}$.

However, equations (132)-(138) are equivalent to those found by entirely different processes by Darboux* for the transformation of isothermic surfaces which he discovered and to which Bianchi has given the name transformations $D_{m}$.

In consequence of the foregoing results we have

Theorem 14. If two surfaces $S$ and $S_{1}$ in the relation of a transformation $K$ constitute the envelope of a two-parameter family of spheres, the surfaces are isothermic and $S_{1}$ is obtained from $S$ by a transformation $D_{m}$.

In accordance with (136) we define a function $\phi_{1}$ by

$$
e^{\phi_{1}}=\frac{e^{-\phi} \theta}{\lambda}
$$

If $X_{1}^{\prime}, Y_{1}^{\prime}, Z_{1}^{\prime}$ and $X_{2}^{\prime}, Y_{2}^{\prime}, Z_{2}^{\prime}$ denote the direction-cosines of the tangents to the parametric curves on $S_{1}$, it follows from (135) and (137) that

$$
\begin{aligned}
& X_{1}^{\prime}=-\frac{1}{m \lambda \theta}\left[\left(\alpha^{2}+\theta \lambda m\right) X_{1}+\alpha \beta X_{2}+\alpha w X\right], \\
& X_{2}^{\prime}=\frac{1}{m \lambda \theta}\left[\alpha \beta X_{1}+\left(\beta^{2}+\theta \lambda m\right) X_{2}+\beta w X\right] .
\end{aligned}
$$

On replacing $X_{1}$ and $X_{2}$ in (105) and (110) by $\left(X_{1}+X_{2}\right) / \sqrt{2}$ and $\left(-X_{1}+X_{2}\right) / \sqrt{2}$ respectively, we have the first derivatives of $X, X_{1}$, and $X_{2}$. With the aid of these formulas we obtain from (140)

$$
\begin{aligned}
& \frac{\partial X^{\prime}}{\partial u}=\left[\frac{e^{\phi}}{\rho_{1}}+\frac{w}{\theta \lambda}\left(\lambda e^{\phi}-\theta e^{-\phi}\right)\right] X_{1}^{\prime}, \\
& \frac{\partial X^{\prime}}{\partial v}=-\left[\frac{e^{\phi}}{\rho_{2}}+\frac{w}{\theta \lambda}\left(\lambda e^{\phi}+\theta e^{-\phi}\right)\right] X_{2}^{\prime} .
\end{aligned}
$$

From these expressions and (135) it follows that the principal radii $\rho_{1}^{\prime}$ and $\rho_{2}^{\prime}$ of $S_{1}$ are given by

* Loc. cit., p. 502.

$$
\begin{aligned}
& \frac{e^{\phi_{1}}}{\rho_{1}^{\prime}}=-\left[\frac{e^{\phi}}{\rho_{1}}+\frac{w}{\theta \lambda}\left(\lambda e^{\phi}-\theta e^{-\phi}\right)\right], \\
& \frac{e^{\phi_{1}}}{\rho_{2}^{\prime}}=\left[\frac{e^{\phi}}{\rho_{2}}+\frac{w}{\theta \lambda}\left(\lambda e^{\phi}+\theta e^{-\phi}\right)\right] .
\end{aligned}
$$


10. Theorem of permutability of transformations $D_{m}$

In this section we apply the results of $\S 3$ to an immediate proof of the theorem of permutability of transformations $D_{m}$ which Bianchi* established at length by direct processes. We are thus able to appreciate the underlying reason for the existence of this theorem.

In the first place we seek solutions $\alpha_{1}^{\prime}, \beta_{1}^{\prime}, w_{1}^{\prime}, \theta_{1}^{\prime}, \lambda_{1}^{\prime}$ of equations for $S_{1}$ analogous to (132) and (133), it being understood that two sets of solutions of the latter equations are known, which we denote by $\alpha_{i}, \beta_{i}, w_{i}, \theta_{i}, \lambda_{i}$ for $i=1,2$. The functions $\theta_{1}^{\prime}$ and $\lambda_{1}^{\prime}$ are given by equations (27) and (36). Hence if $\alpha_{1}^{\prime}$ and $\beta_{1}^{\prime}$ are to satisfy

$$
\frac{\partial \theta_{1}^{\prime}}{\partial u}=e^{\phi_{1}} \alpha_{1}^{\prime}=-\frac{e^{-\phi} \theta_{1}}{\lambda_{1}} \alpha_{1}^{\prime}, \quad \frac{\partial \theta_{1}^{\prime}}{\partial v}=-\frac{e^{-\phi} \theta_{1}}{\lambda_{1}} \beta_{1}^{\prime},
$$

we must have

$$
\begin{gathered}
\alpha_{1}^{\prime}=-\frac{\alpha_{1}}{\theta_{1}}\left(\theta_{1}^{\prime}+\theta_{2}\right)+\alpha_{2}, \quad \beta_{1}^{\prime}=\frac{\beta_{1}}{\theta_{1}}\left(\theta_{1}^{\prime}+\theta_{2}\right)-\beta_{2}, \\
\lambda_{1}^{\prime}=\frac{\lambda_{1}}{\theta_{1}}\left(\theta_{1}^{\prime}+\theta_{2}\right)-\lambda_{2} .
\end{gathered}
$$

These values of $\alpha_{1}^{\prime}$ and $\beta_{1}^{\prime}$ satisfy identically the equations

$$
\frac{\partial \alpha_{1}^{\prime}}{\partial v}=\beta_{1}^{\prime} \frac{\partial \phi_{1}}{\partial u}, \quad \frac{\partial \beta_{1}^{\prime}}{\partial u}=\alpha_{1}^{\prime} \frac{\partial \phi_{1}}{\partial v},
$$

and when substituted in

$$
\begin{aligned}
& \frac{\partial \alpha_{1}^{\prime}}{\partial u}=-m_{1}^{\prime} \lambda_{1}^{\prime} e^{\phi_{1}}+m_{1}^{\prime} e^{-\phi_{1}} \theta_{1}^{\prime}-\frac{\partial \phi_{1}}{\partial v} \beta_{1}^{\prime}+w_{1}^{\prime} \frac{e^{\phi_{1}}}{\rho_{1}^{\prime}}, \\
& \frac{\partial \beta_{1}^{\prime}}{\partial v}=-m_{1}^{\prime} \lambda_{1}^{\prime} e^{\phi_{1}}-m_{1}^{\prime} e^{-\phi_{1}} \theta_{1}^{\prime}-\frac{\partial \phi_{1}}{\partial u} \alpha_{1}^{\prime}+w_{1}^{\prime} \frac{\phi_{1}}{\rho_{2}^{\prime}},
\end{aligned}
$$

and use is made of the foregoing equations, we get

$$
\begin{aligned}
& A \frac{e^{\phi_{1}}}{\rho_{1}^{\prime}}+\left(\theta_{1} e^{-\phi}-\lambda_{1} e^{\phi}\right) B+\lambda_{1} e^{\phi}\left(m_{2} \frac{\lambda_{2}}{\lambda_{1}}+m_{1}^{\prime} \frac{\theta_{2}}{\theta_{1}}\right)-\theta_{1} e^{-\phi}\left(m_{1}^{\prime} \frac{\lambda_{2}}{\lambda_{1}}+m_{2} \frac{\theta_{2}}{\theta_{1}}\right)=0, \\
& A \frac{e^{\phi_{1}}}{\rho_{2}^{\prime}}+\left(\theta_{1} e^{-\phi}+\lambda_{1} e^{\phi}\right) B-\lambda_{1} e^{\phi}\left(m_{2} \frac{\lambda_{2}}{\lambda_{1}}+m_{1}^{\prime} \frac{\theta_{2}}{\theta_{1}}\right) \\
&-\theta_{1} e^{-\phi}\left(m_{1}^{\prime} \frac{\lambda_{2}}{\lambda_{1}}+m_{2} \frac{\theta_{2}}{\theta_{1}}\right)=0,
\end{aligned}
$$

* Loc. cit., pp. 109-125. 
where

$$
A=w_{1}^{\prime}-\frac{w_{1}}{\theta_{1}}\left(\theta_{1}^{\prime}+\theta_{2}\right)+w_{2},
$$

$$
B=\frac{1}{\theta_{1}}\left[\left(\theta_{1}^{\prime}+\theta_{2}\right)\left(m_{1}^{\prime}-m_{1}\right)-\frac{1}{\lambda_{1}}\left(\alpha_{1} \alpha_{2}+\beta_{1} \beta_{2}+\gamma_{1} \gamma_{2}\right)\right] .
$$

If we put $m_{1}^{\prime}=m_{2}$, these equations become

$$
A \frac{e^{\phi_{1}}}{\rho_{1}^{\prime}}+\frac{C}{\theta_{1}}\left(\theta_{1} e^{-\phi}-\lambda_{1} e^{\phi}\right)=0, \quad A \frac{e^{\phi_{1}}}{\rho_{2}^{\prime}}+\frac{C}{\theta_{1}}\left(\theta_{1} e^{-\phi}+\lambda_{1} e^{\phi}\right)=0,
$$

where

$$
\begin{aligned}
C=\left(\theta_{1}^{\prime}+\theta_{2}\right)\left(m_{2}-m_{1}\right)-\frac{1}{\lambda_{1}}\left(\alpha_{1} \alpha_{2}+\beta_{1} \beta_{2}\right. & \left.+w_{1} w_{2}\right) \\
& -\frac{m_{2}}{\lambda_{1}}\left(\lambda_{2} \theta_{1}+\lambda_{1} \theta_{2}\right) .
\end{aligned}
$$

Equations (147) are satisfied when $A=C=0$. From these equations and (144), we have

$$
\begin{aligned}
-\alpha_{1}^{\prime} & =\kappa\left[\frac{\Phi_{1} \alpha_{1}}{\theta_{1} \lambda_{1}}+\left(m_{1}-m_{2}\right) \alpha_{2}\right], \\
\beta_{1}^{\prime} & =\kappa\left[\frac{\Phi_{1} \beta_{1}}{\theta_{1} \lambda_{1}}+\left(m_{1}-m_{2}\right) \beta_{2}\right], \\
w_{1}^{\prime} & =\kappa\left[\frac{\Phi_{1} w_{1}}{\theta_{1} \lambda_{1}}+\left(m_{1}-m_{2}\right) w_{2}\right], \\
\lambda_{1}^{\prime} & =\kappa\left[\frac{\Phi_{1}}{\theta_{1}}+\left(m_{1}-m_{2}\right) \lambda_{2}\right], \quad \kappa=\frac{1}{m_{2}-m_{1}}, \\
\theta_{1}^{\prime} & =\kappa\left[\frac{\Phi_{1}}{\lambda_{1}}+\left(m_{1}-m_{2}\right) \theta_{2}\right], \quad
\end{aligned}
$$

where we have put

$$
\Phi_{1}=\alpha_{1} \alpha_{2}+\beta_{1} \beta_{2}+\gamma_{1} \gamma_{2}+m_{2}\left(\lambda_{2} \theta_{1}+\lambda_{1} \theta_{2}\right) \text {. }
$$

It is readily shown that these values of $\theta_{1}^{\prime}$ and $w_{1}^{\prime}$ satisfy equations (143) and also

$$
\frac{\partial w_{1}^{\prime}}{\partial u}=-\alpha_{1}^{\prime} \frac{e^{\phi_{1}}}{\rho_{1}^{\prime}}, \quad \frac{\partial w_{1}^{\prime}}{\partial v}=-\beta_{1}^{\prime} \frac{e^{\phi_{1}}}{\rho_{2}^{\prime}},
$$

and consequently the functions (149) satisfy all the conditions necessary to the problem. Hence we have established with Bianchi

Theorem 15. If $S$ is an isothermic surface, and two isothermic surfaces $S_{1}$ and $S_{2}$ are obtained from $S$ by transformations $D_{m 1}$ and $D_{m 2}$, there exists a 
transformation $D_{m 2}^{\prime}$ of $S_{1}$ into a surface $S^{\prime}$ which can be obtained from $S_{2}$ also by a transformation $D_{m 1}^{\prime}$; moreover, $S^{\prime}$ can be found without quadratures.

When $S$ is isothermic, the associate surface defined by equations (11) is the Christoffel transform. Hence, as an immediate consequence of Theorem 5, we have that transformations $D_{m}$ are commutative with transformations of Christoffel, a result which Bianchi established by direct calculation.

\section{Congruences $(G)$ with more than one pair of surfaces in the RELATION OF A TRANSFORMATION $K$}

In this section we consider the consistency of equations (13) and (18). We write the former in the form of the second of equations (15) and express the condition of integrability. This gives

$$
\frac{\partial M}{\partial v}=\frac{k}{N^{2}} k_{-1}+M \frac{\partial \log k}{\partial v}+2 M^{2}-\frac{3 M k}{N},
$$

where $k_{-1}$, given by

$$
k_{-1}=2 k-h-\frac{\partial^{2} \log k}{\partial u \partial v},
$$

is an invariant of the point equation of the second focal surface of tangents to the curves $u=$ const. on $S_{0}$.

The condition of integrability of (151) and of the first of (15) can be reduced to

$$
N^{2} h \frac{\partial}{\partial v} \log \frac{h}{k}+\left(k_{-1} \frac{\partial k}{\partial u}-k \frac{\partial k_{-1}}{\partial u}\right)+3 N(M N-k)\left(k_{-1}-h\right)=0 .
$$

Hence the necessary and sufficient condition that (151) and (15) be consistent identically is that

$$
k_{-1}=h=(c+1) k,
$$

where $c$ denotes an arbitrary constant. In this case it follows from (152) and (154) that

$$
\frac{\partial^{2} \log k}{\partial u \partial v}+2 c k=0 .
$$

When $c=0$, we have

$$
h=k=U V,
$$

where $U$ and $V$ are arbitrary functions of $u$ and $v$ respectively. When $c \neq 0$, the general integral of (155) is

$$
\dot{k}=\frac{U^{\prime} V^{\prime}}{c(1+U V)^{2}}, \quad h=(c+1) k,
$$

$U$ and $V$ being arbitrary functions of $u$ and $v$ respectively and a prime indicating differentiation with respect to the argument. 
When $c=-1, h=0$ and it is readily shown that in this case $S$ and $S_{1}$ coincide. Consequently we exclude this value.

If we take the expressions (156) or (157) for $h$ and $k$, and determine $a$ and $b$ in accordance with $\left(14^{\prime}\right)$ two arbitrary functions are introduced. Then each of the functions $M$ and $N$ as determined by equations (15), (18), and (151) involves an arbitrary constant, and $\sigma$ and $\sigma_{1}$ are found by quadratures. In view of these results we have

TheOREM 16. Whenever the point equation of a surface $S_{0}$ has invariants of the form (156) or (157), there exist on the tangents to the curves $v=$ const. of $S_{0}$ an infinity of pairs of points which generate pairs of surfaces in the relations of transformations $K$; the same is true of the tangents to the curves $u=$ const. of $S_{0}$, if $h=k=U V$.

Returning to the case when equation (153) is not satisfied identically, we differentiate this equation respectively with respect to $u$ and $v$, and replace the derivatives of $M$ and $N$ by their expressions from (15), (18), and (151). By means of (153) the two resulting equations are reducible to the forms

$$
P N^{2}+Q=0, \quad R N^{2}+S=0,
$$

where $P, Q, R, S$ are determinate functions involving $h, k, k_{-1}$ and their derivatives. When we express the conditions that these equations be consistent, and that the expressions for $N$ thus given and of $M$ from (153) shall satisfy equations (15), (18), (151), we obtain the equations for $h$ and $k$ which determine the surfaces $S_{0}$. We are not interested in these equations but in the fact that from the form of equations (158) and (16) it follows that there is only one pair of surfaces $S$ and $S_{1}$ associated with the given congruence. Hence we have

THEOREM 17. If a congruence $(G)$ possesses more than one pair of points which generate surfaces in the relation of a transformation $K$, it possesses an infinity of such pairs.

Princeton University, April 14, 1914. 